Research Article

\title{
The Sub-Riemannian Limit of Curvatures for Curves and Surfaces and a Gauss-Bonnet Theorem in the Rototranslation Group
}

\author{
Haiming Liu $\mathbb{D}$, Jiajing Miao, Wanzhen Li, and Jianyun Guan \\ School of Mathematics, Mudanjiang Normal University, Mudanjiang 157011, China \\ Correspondence should be addressed to Haiming Liu; haiming0626@126.com
}

Received 21 March 2021; Revised 3 May 2021; Accepted 19 May 2021; Published 3 June 2021

Academic Editor: Antonio Masiello

Copyright (c) 2021 Haiming Liu et al. This is an open access article distributed under the Creative Commons Attribution License, which permits unrestricted use, distribution, and reproduction in any medium, provided the original work is properly cited.

\begin{abstract}
The rototranslation group $\mathscr{R T}$ is the group comprising rotations and translations of the Euclidean plane which is a 3-dimensional Lie group. In this paper, we use the Riemannian approximation scheme to compute sub-Riemannian limits of the Gaussian curvature for a Euclidean $C^{2}$-smooth surface in the rototranslation group away from characteristic points and signed geodesic curvature for Euclidean $C^{2}$-smooth curves on surfaces. Based on these results, we obtain a Gauss-Bonnet theorem in the rototranslation group.
\end{abstract}

\section{Introduction}

The Gauss-Bonnet theorem connects the intrinsic differential geometry of a surface with its topology and has many applications in physics and mathematics. For example, Petters used the Gauss-Bonnet theorem to study the global geometry of caustics for multiple lens planes in the impulse approximation [1]. Gibbons and Werner showed that it is possible to calculate the deflection angle in weak field limits using the Gauss-Bonnet theorem and the optical geometry [2]. In this method, they found that the focusing of the light rays emerges as a topological effect. In 2018, Övgün et al. used the Gauss-Bonnet theorem to obtain the deflection angle by the photons coupled to the Weyl tensor in a Schwarzschild black hole and Schwarzschild-like black hole in bumblebee gravity in the weak limit approximation [3]. Their computations about the weak gravitational lensing of the Kerr-MOG black hole utilized the method of Gauss-Bonnet first prescribed by Gibbons and Werner [2], which reveals the ignored role of topology in gravitational lensing. In 2019, they studied the weak gravitational lensing by the Kerr-MOG black hole and showed that the MOG effect could be taken into account in the gravitational lensing experiment [4]. In 2020, they applied the RVB method, which considers the topological fractions together with the
Gauss-Bonnet theorem and different spacetimes including the nonasymptotically flat ones. This approach showed that Hawking radiation possesses a topological effect coming from the Euler characteristic of the spacetime. The Ricci scalar of the spacetime encodes all the information about the spacetime, which means that it can derive the temperature of the black hole with the Euler characteristic of the metric [5]. They also employed the Gauss-Bonnet theorem to compute the deflection angle by a NAT black hole in the weak limit approximation [6]. In 2021, Chen et al. investigated the photon sphere, shadow, and QNMs of the four-dimensional charged Einstein-Gauss-Bonnet black hole [7]. In this paper, we focus on the Gauss-Bonnet theorem in the rototranslation group.

The rototranslation group, $\mathscr{R T}$, is the group of Euclidean rotations and translations of the plane equipped with a particular sub-Riemannian metric. More precisely, $\mathscr{R T}$ is a three-dimensional topological manifold diffeomorphic to $\mathbb{R}^{2} \times S^{1}$ with coordinates $(x, y, \theta)$. The sub-Riemannian geometry of the rototranslation group is in contrast to the well-known case of the Heisenberg group in mathematics, and it provides geometrical models in mechanics and robotics $[8,9]$. In [10], the rototranslation group $\mathscr{R} \mathscr{T}$ and its universal cover $\mathscr{G}$ were introduced. The main theorem states that a straight ruled surface in $\mathscr{G}$ is horizontally minimal. 
Among more recent works, data representations in orientation scores as a function on the rototranslation group have been used for template matching with cross-correlation [11]. Bekkers et al. recognized a curved geometry on the positionorientation domain, which they identified with the rototranslation group. Templates were then optimized in a B-spline basis, and smoothness was defined with respect to the curved geometry. In [12], illusory patterns were identified by a suitable modulation of the geometry of the rototranslation group and computed as the associated geodesics via the fast marching algorithm. In [13, 14], Balogh et al. used a Riemannian approximation scheme to define a notion of the intrinsic Gaussian curvature for a Euclidean $C^{2}$-smooth surface in the Heisenberg group $H^{1}$ away from characteristic points and a notion of the intrinsic signed geodesic curvature for Euclidean $C^{2}$-smooth curves on surfaces. These results were then used to prove a Heisenberg version of the Gauss-Bonnet theorem. They proposed an interesting question to understand to what extent similar phenomena hold in other sub-Riemannian geometric structures. In [15-17], Wang and Wei solved this problem for the affine group, the group of rigid motions of the Minkowski plane, the BCV spaces, the twisted Heisenberg group, and the Lorentzian Heisenberg group. Their approach is to define sub-Riemannian objects as limits of horizontal objects in $\left(\mathbb{G}, g_{L}\right)$, where a family of metrics $g_{L}$ is essentially obtained as an anisotropic blowup of the Riemannian metric $g_{1}$. At the heart of this approach is the fact that the intrinsic horizontal geometry does not change with $L$. In this paper, we try to solve the above problem for the rototranslation group. We compute sub-Riemannian limits of the Gaussian curvature for a Euclidean $C^{2}$-smooth surface in the rototranslation group away from characteristic points and signed geodesic curvature for Euclidean $C^{2}$-smooth curves on surfaces. We also obtain a Gauss-Bonnet theorem in the rototranslation group.

In Section 2, we provide a short introduction to the rototranslation group and the notion which we will use throughout the paper, such as the Levi-Civita connection and curvature in the Riemannian approximants of the rototranslational group. In Section 3, we compute the subRiemannian limit of the curvature of curves in the rototranslation group. In Sections 4 and 5, we compute subRiemannian limits of the geodesic curvature of curves on surfaces and the Riemannian Gaussian curvature of surfaces in the rototranslation group. In Section 6, we obtain the Gauss-Bonnet theorem in the rototranslation group. In Section 7 , we summarize this paper as conclusions.

\section{Levi-Civita Connection and Curvature in the Riemannian Approximants of the Rototranslation Group}

In this section, some basic notions in the rototranslation group will be introduced. The rototranslation group $\mathscr{R} \mathscr{T}$ is the group comprising rotations and translations of the Euclidean plane. It is a 3-dimensional Lie group, isomorphic to $\mathbb{R}^{2} \times S^{1}$ with multiplication given by

$$
(x, y, \theta) *\left(x^{\prime}, y^{\prime}, \theta^{\prime}\right)=\left(x+x^{\prime} \cos \theta-y^{\prime} \sin \theta, y+x^{\prime} \sin \theta+y^{\prime} \cos \theta, \theta+\theta^{\prime}\right)
$$

for all $(x, y, \theta),\left(x^{\prime}, y^{\prime}, \theta^{\prime}\right) \in \mathbb{R}^{2} \times S^{1}$. In this model, the natural element of $\mathscr{R} \mathscr{T}$ is $(0,0,0)$, and the inverse element of $(x, y, \theta)$ is $(-x \cos \theta-y \sin \theta, x \sin \theta-y \cos \theta,-\theta)$.

Let

$$
\begin{aligned}
& X_{1}=\cos \theta \frac{\partial}{\partial x}+\sin \theta \frac{\partial}{\partial y} \\
& X_{2}=\frac{\partial}{\partial \theta} \\
& X_{3}=\sin \theta \frac{\partial}{\partial x}-\cos \theta \frac{\partial}{\partial y}
\end{aligned}
$$

with brackets

$$
\begin{aligned}
& {\left[X_{1}, X_{2}\right]=X_{3},} \\
& {\left[X_{2}, X_{3}\right]=X_{1},} \\
& {\left[X_{1}, X_{3}\right]=0}
\end{aligned}
$$

Then,

$$
\begin{aligned}
\frac{\partial}{\partial x} & =\cos \theta X_{1}+\sin \theta X_{3}, \\
\frac{\partial}{\partial y} & =\sin \theta X_{1}-\cos \theta X_{3}, \\
\frac{\partial}{\partial \theta} & =X_{2},
\end{aligned}
$$

and $\operatorname{span}\left\{X_{1}, X_{2}, X_{3}\right\}=T(\mathscr{R T})$. Let $H=\operatorname{span}\left\{X_{1}, X_{2}\right\}$ be the horizontal distribution on $\mathscr{R} \mathscr{T}$. Let $\omega_{1}=\cos$ $\theta \mathrm{d} x+\sin \theta \mathrm{d} y, \omega_{2}=\mathrm{d} \theta$, and $\omega=\sin \theta \mathrm{d} x-\cos \theta \mathrm{d} y$. Then, $H=\operatorname{ker} \omega$. To describe the Riemannian approximants to $\mathscr{R} \mathscr{T}$, for the constant $L>0$, we define a metric $g_{L}=\omega_{1} \otimes \omega_{1}+\omega_{2} \otimes \omega_{2}+L \omega \otimes \omega$ so that $X_{1}, X_{2}, \widetilde{X_{3}}$ $:=L^{-(1 / 2)} X_{3}$ are orthonormal bases on $T(\mathscr{R} \mathscr{T})$ with respect to $g_{L}$. It is easy to check that $g=g_{1}$ is the Riemannian metric on $\mathscr{R} \mathscr{T}$.

To compute the sectional, Ricci, and scalar curvatures of the rototranslation group with respect to $g_{L}$, we use the LeviCivita connection $\nabla^{L}$ on $\left(\mathscr{R} \mathscr{T}, g_{L}\right)$. A straightforward calculation shows the following proposition. 
Proposition 1. Let $\mathscr{R} \mathscr{T}$ be the rototranslation group, relative to the coordinate frame $X_{1}, X_{2}, \widetilde{X_{3}}$; then, the Levi-Civita connection on $\mathscr{R} \mathscr{T}$ is given by

$$
\begin{aligned}
& \nabla_{X_{j}}^{L} X_{j}=0, \quad 1 \leq j \leq 3, \\
& \nabla_{X_{1}}^{L} X_{2}=\frac{L-1}{2 L} X_{3}, \\
& \nabla_{X_{2}}^{L} X_{1}=\frac{-(L+1)}{2 L} X_{3}, \\
& \nabla_{X_{1}}^{L} X_{3}=\frac{1-L}{2} X_{2}, \\
& \nabla_{X_{3}}^{L} X_{1}=\frac{(-L+1)}{2} X_{2},
\end{aligned}
$$

$$
\begin{aligned}
& \nabla_{X_{2}}^{L} X_{3}=\frac{1+L}{2} X_{1}, \\
& \nabla_{X_{3}}^{L} X_{2}=\frac{-1+L}{2} X_{1} .
\end{aligned}
$$

Proof. It follows from a direct application of the Koszul identity, which here simplifies

$$
\begin{aligned}
2\left\langle\nabla_{X_{i}}^{L} X_{j}, X_{k}\right\rangle_{L}= & \left\langle\left[X_{i}, X_{j}\right], X_{k}\right\rangle_{L}-\left\langle\left[X_{j}, X_{k}\right], X_{i}\right\rangle_{L} \\
& +\left\langle\left[X_{k}, X_{i}\right], X_{j}\right\rangle_{L},
\end{aligned}
$$

where $i, j, k=1,2,3$. By (3) and (6), we have

$$
\begin{aligned}
2\left\langle\nabla_{X_{j}}^{L} X_{j}, X_{k}\right\rangle_{L} & =\left\langle\left[X_{j}, X_{j}\right], X_{k}\right\rangle_{L}-\left\langle\left[X_{j}, X_{k}\right], X_{j}\right\rangle_{L}+\left\langle\left[X_{k}, X_{j}\right], X_{j}\right\rangle_{L} \\
& =-\left\langle\left[X_{j}, X_{k}\right], X_{j}\right\rangle_{L}+\left\langle\left[X_{k}, X_{j}\right], X_{j}\right\rangle_{L}=2\left\langle\left[X_{k}, X_{j}\right], X_{j}\right\rangle_{L}
\end{aligned}
$$

When $j=1$, we compute $\left\langle\nabla_{X_{1}}^{L} X_{1}, X_{k}\right\rangle_{L}=\left\langle\left[X_{k}\right.\right.$, $\left.\left.X_{1}\right], X_{1}\right\rangle_{L}$. It follows that $\left\langle\nabla_{X_{1}}^{L} X_{1}, X_{1}\right\rangle_{L}=0,\left\langle\nabla_{X_{1}}^{L} X_{1}, X_{2}\right\rangle_{L}$ $=\left\langle\left[X_{2}, X_{1}\right], X_{1}\right\rangle_{L}=\left\langle-X_{3}, X_{1}\right\rangle_{L}=0$, and $\left\langle\nabla_{X_{1}}^{L_{1}} X_{1}, X_{3}\right\rangle_{L}$ $=\left\langle\left[X_{3}, X_{1}\right], X_{1}\right\rangle_{L}=0$. Hence, $\nabla_{X_{1}}^{L} X_{1}=0$. Similarly, $\nabla_{X_{2}}^{L} X_{2}=0$ and $\nabla_{X_{3}}^{L} X_{3}=0$. By the following equation,

$$
\begin{aligned}
2\left\langle\nabla_{X_{1}}^{L} X_{2}, X_{k}\right\rangle_{L}= & \left\langle\left[X_{1}, X_{2}\right], X_{k}\right\rangle_{L}-\left\langle\left[X_{2}, X_{k}\right], X_{1}\right\rangle_{L} \\
& +\left\langle\left[X_{k}, X_{1}\right], X_{2}\right\rangle_{L}=\left\langle X_{3}, X_{k}\right\rangle_{L} \\
& -\left\langle\left[X_{2}, X_{k}\right], X_{1}\right\rangle_{L}+\left\langle\left[X_{k}, X_{1}\right], X_{2}\right\rangle_{L}
\end{aligned}
$$

we get $\nabla_{X_{1}}^{L} X_{2}=(L-1 / 2 L) X_{3}$. Other cases follow the similar way.

Define the curvature of the connection $\nabla^{L}$ by

$$
R^{L}(X, Y) Z=\nabla_{X}^{L} \nabla_{Y}^{L} Z-\nabla_{Y}^{L} \nabla_{X}^{L} Z-\nabla_{[X, Y]}^{L} Z .
$$

We get the following proposition.

Proposition 2. Let $\mathscr{R} \mathscr{T}$ be the rototranslation group; then,

$$
\begin{aligned}
& R^{L}\left(X_{1}, X_{2}\right) X_{1}=\frac{3 L^{2}-2 L-1}{4 L} X_{2}, \\
& R^{L}\left(X_{1}, X_{2}\right) X_{2}=\frac{-3 L^{2}+2 L+1}{4 L} X_{1}, \\
& R^{L}\left(X_{1}, X_{2}\right) X_{3}=0, \\
& R^{L}\left(X_{1}, X_{3}\right) X_{1}=\frac{-L^{2}+2 L-1}{4 L} X_{3}, \\
& R^{L}\left(X_{1}, X_{3}\right) X_{2}=0, \\
& R^{L}\left(X_{1}, X_{3}\right) X_{3}=\frac{L^{2}-2 L+1}{4} X_{1},
\end{aligned}
$$

$$
\begin{aligned}
& R^{L}\left(X_{2}, X_{3}\right) X_{1}=0, \\
& R^{L}\left(X_{2}, X_{3}\right) X_{2}=\frac{-L^{2}-2 L+3}{4 L} X_{3}, \\
& R^{L}\left(X_{2}, X_{3}\right) X_{3}=\frac{L^{2}+2 L-3}{4} X_{2} .
\end{aligned}
$$

Proof. It is a direct computation using

$$
R^{L}(X, Y) Z=\nabla_{X}^{L} \nabla_{Y}^{L} Z-\nabla_{Y}^{L} \nabla_{X}^{L} Z-\nabla_{[X, Y]}^{L} Z .
$$

\section{Taking}

$$
R^{L}\left(X_{1}, X_{2}\right) X_{1}=\left(\nabla_{X_{1}}^{L} \nabla_{X_{2}}^{L}-\nabla_{X_{2}}^{L} \nabla_{X_{1}}^{L}-\nabla_{\left[X_{1}, X_{2}\right]}^{L}\right) X_{1} \text {, }
$$

for example, we compute

$$
\begin{aligned}
& \nabla_{X_{1}}^{L}\left(\nabla_{X_{2}}^{L} X_{1}\right)=\frac{-(L+1)}{2 L} \nabla_{X_{1}}^{L} X_{3}=\frac{L^{2}-1}{4 L} X_{2}, \\
& \nabla_{X_{2}}^{L}\left(\nabla_{X_{1}}^{L} X_{1}\right)=0, \\
& \nabla_{\left[X_{1}, X_{2}\right]}^{L} X_{1}=\nabla_{X_{3}}^{L} X_{1}=\frac{(-L+1)}{2} X_{2} .
\end{aligned}
$$

Hence,

$$
R^{L}\left(X_{1}, X_{2}\right) X_{1}=\frac{L^{2}-1}{4 L} X_{2}-\frac{(-L+1)}{2} X_{2}=\frac{3 L^{2}-2 L-1}{4 L} X_{2} .
$$

We compute the sectional curvatures of the two planes spanned by the basis vectors $\tilde{X}_{i}$ and $\tilde{X}_{j}$ : 
$\mathscr{K}_{i j}=\left\langle R^{L}\left(\widetilde{X}_{i}, \widetilde{X}_{j}\right) \widetilde{X}_{i}, \widetilde{X}_{j}\right\rangle_{L}$, where $\widetilde{X}_{i}=X_{i}$ for $i=1,2$ and $\widetilde{X}_{3}=L^{-(1 / 2)} X_{3}$.

$$
\begin{aligned}
& \mathscr{K}_{12}=\left\langle R^{L}\left(\widetilde{X}_{1}, \widetilde{X}_{2}\right) \widetilde{X}_{1}, \widetilde{X}_{2}\right\rangle_{L}=\frac{3 L^{2}-2 L-1}{4 L}, \\
& \mathscr{K}_{13}=\left\langle R^{L}\left(\widetilde{X}_{1}, \widetilde{X}_{3}\right) \widetilde{X}_{1}, \widetilde{X}_{3}\right\rangle_{L}=\frac{-L^{2}+2 L-1}{4 L}, \\
& \mathscr{K}_{23}=\left\langle R^{L}\left(\widetilde{X}_{2}, \widetilde{X}_{3}\right) \widetilde{X}_{2}, \widetilde{X}_{3}\right\rangle_{L}=\frac{-L^{2}-2 L+3}{4 L} .
\end{aligned}
$$

In fact, the full Riemannian curvature tensor $R_{i j k l}^{L}=\left\langle R^{L}\left(\widetilde{X}_{i}, \widetilde{X}_{j}\right) \widetilde{X}_{k}, \widetilde{X}_{l}\right\rangle_{L}$ is

$$
R_{i j k l}^{L}= \begin{cases}\frac{3 L^{2}-2 L-1}{4 L} & \text { if }(i j k l)=(1212) \text { or }(2121), \\ \frac{-3 L^{2}+2 L+1}{4 L} & \text { if }(i j k l)=(1221) \text { or }(2112), \\ \frac{-L^{2}+2 L-1}{4 L} & \text { if }(i j k l)=(1313),(3131),(2323) \text { or }(3232), \\ \frac{L^{2}-2 L+1}{4 L} & \text { if }(i j k l)=(1331),(3113),(2332) \text { or }(3223), \\ 0, & \text { otherwise. }\end{cases}
$$

In order to compute the Kretschmann scalar, from $R_{L}^{i j k l}=g^{l l} g^{k k} g^{j j} g^{i i} R_{i j k l}^{L}$, it follows that we can write

$$
R_{L}^{i j k l}= \begin{cases}\frac{3 L^{2}-2 L-1}{4 L} & \text { if }(i j k l)=(1212) \text { or }(2121), \\ \frac{-3 L^{2}+2 L+1}{4 L} & \text { if }(i j k l)=(1221) \text { or }(2112), \\ \frac{-L^{2}+2 L-1}{4 L} & \text { if }(i j k l)=(1313),(3131),(2323) \text { or }(3232), \\ \frac{L^{2}-2 L+1}{4 L} & \text { if }(i j k l)=(1331),(3113),(2332) \text { or }(3223), \\ 0, & \text { otherwise. }\end{cases}
$$


Recall that the Kretschmann scalar is defined by $\mathscr{K}=R_{i j k l}^{L} R_{L}^{i j k l}$. We calculate that

$$
\begin{aligned}
\mathscr{K} & =4\left[\left(R_{1212}^{L}\right)^{2}+\left(R_{1313}^{L}\right)^{2}+\left(R_{2323}^{L}\right)^{2}\right] \\
& =\frac{11 L^{4}-20 L^{3}+10 L^{2}-4 L+3}{4 L^{2}} .
\end{aligned}
$$

Next, the Ricci curvature $\operatorname{Ric}_{i}=\mathscr{K}_{i 1}+\mathscr{K}_{i 2}+\mathscr{K}_{i 3}$ is

$$
\begin{aligned}
& R i c_{1}=\frac{L}{2}-\frac{1}{2 L}, \\
& R i c_{2}=-L+\frac{1}{2 L}-1, \\
& R i c_{3}=-\frac{L}{2}+\frac{1}{2 L},
\end{aligned}
$$

while the scalar curvature

$$
\sigma=R i c_{1}+R i c_{2}+R i c_{3}=-L+\frac{1}{2 L}-1 .
$$

It can be observed that the Kretschmann scalar and the sectional, Ricci, and scalar curvatures all diverge as $L \longrightarrow \infty$.

\section{The Sub-Riemannian Limit of the$$
\text { Curvature of Curves in the }
$$$$
\text { Rototranslation Group }
$$

In this section, we will compute the sub-Riemannian limit of the curvature of curves in the rototranslation group.
Definition 1. Let $\gamma:[a, b] \longrightarrow\left(\mathscr{R T}, g_{L}\right)$ be a Euclidean $C^{1}$-smooth curve; we say that $\gamma$ is regular if $\dot{\gamma} \neq 0$ for every $t \in[a, b]$. Moreover, we say that $\gamma(t)$ is a horizontal point of $\gamma$ if

$$
\begin{aligned}
\omega(\dot{\gamma}(t)) & =(\sin \theta \mathrm{d} x-\cos \theta \mathrm{d} y)\left(\dot{\gamma}_{1}(t) \frac{\partial}{\partial x}+\dot{\gamma}_{2}(t) \frac{\partial}{\partial y}+\dot{\theta}(t) \frac{\partial}{\partial \theta}\right) \\
& =\dot{\gamma}_{1}(t) \sin \theta-\dot{\gamma}_{2}(t) \cos \theta=0
\end{aligned}
$$

where $\gamma(t)=\left(\gamma_{1}(t), \gamma_{2}(t), \theta(t)\right)$.

Definition 2. Let $\gamma:[a, b] \longrightarrow\left(\mathscr{R} \mathscr{T}, g_{L}\right)$ be a Euclidean $C^{2}$-smooth regular curve in the Riemannian manifold $\left(\mathscr{R} \mathscr{T}, g_{L}\right)$. The curvature $\kappa_{\gamma}^{L}$ of $\gamma$ at $\gamma(t)$ is defined as

$$
\kappa_{\gamma}^{L}:=\sqrt{\frac{\left\|\nabla_{\dot{\gamma}}^{L} \dot{\gamma}\right\|_{L}^{2}}{\|\dot{\gamma}\|_{L}^{4}}-\frac{\left\langle\nabla_{\dot{\gamma}}^{L} \dot{\gamma}, \dot{\gamma}\right\rangle_{L}^{2}}{\|\dot{\gamma}\|_{L}^{6}} .}
$$

Proposition 3. Let $\gamma:[a, b] \longrightarrow\left(\mathscr{R T}, g_{L}\right)$ be a Euclidean $C^{2}$-smooth regular curve in the Riemannian manifold $\left(\mathscr{R} \mathscr{T}, g_{L}\right)$. Then,

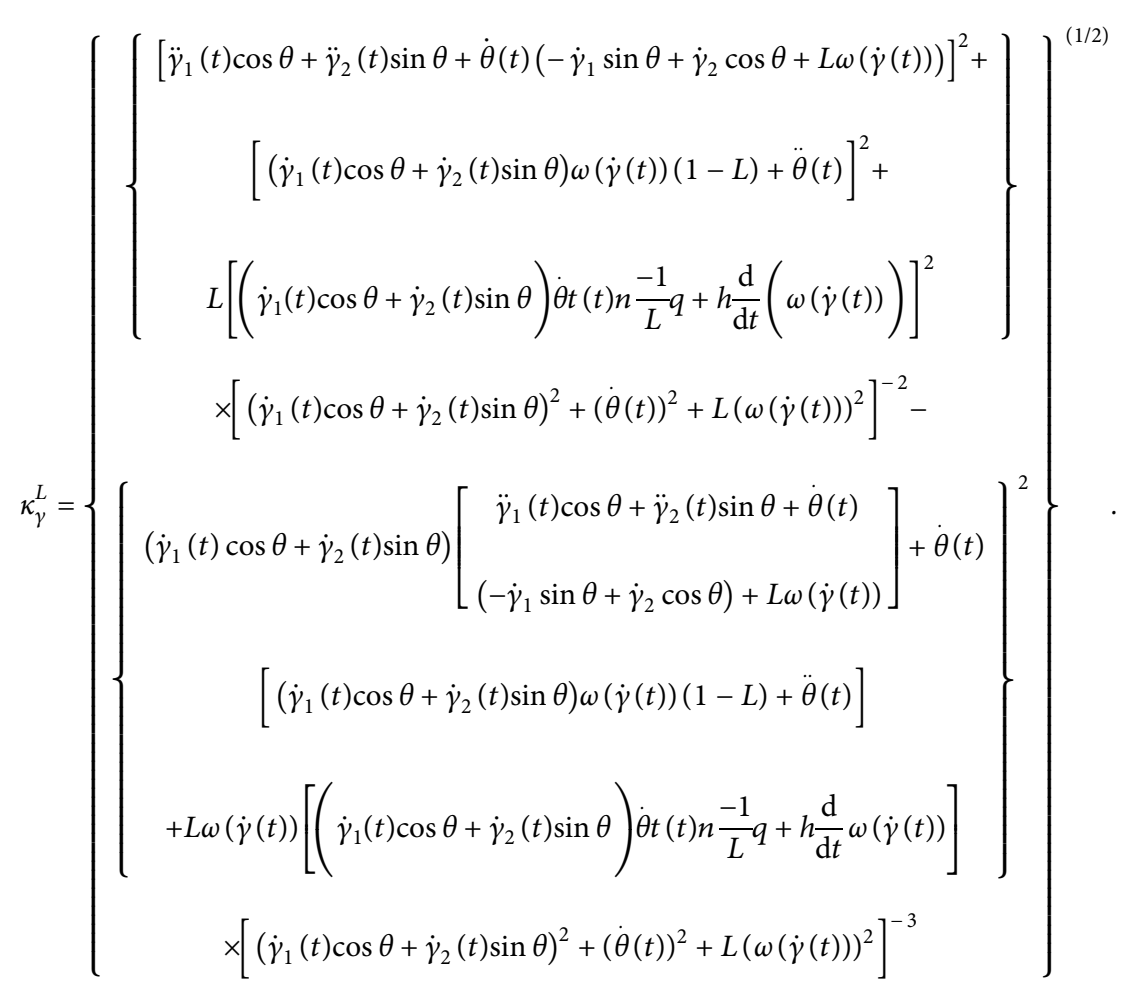


In particular, if $\gamma(t)$ is a horizontal point of $\gamma$,

$$
k_{\gamma}^{L}=\left\{\begin{array}{c}
\left\{\begin{array}{c}
{\left[\ddot{\gamma}_{1}(t) \cos \theta+\ddot{\gamma}_{2}(t) \sin \theta+\dot{\theta}(t)\left(-\dot{\gamma}_{1} \sin \theta+\dot{\gamma}_{2} \cos \theta\right)\right]^{2}+(\ddot{\theta}(t))^{2}} \\
\left.+L\left[\left(\dot{\gamma}_{1}(t) \cos \theta+\dot{\gamma}_{2}(t) \sin \theta\right) \dot{\theta} t(t) n \frac{-1}{L} q+h \frac{\mathrm{d}}{\mathrm{d} t}(\omega(\dot{\gamma}(t)))\right]\right]^{2}
\end{array}\right\} \\
\cdot\left[\left(\dot{\gamma}_{1}(t) \cos \theta+\dot{\gamma}_{2}(t) \sin \theta\right)^{2}+(\dot{\theta}(t))^{2}\right]^{-2}- \\
\left\{\begin{array}{c}
\left(\dot{\gamma}_{1}(t) \cos \theta+\dot{\gamma}_{2}(t) \sin \theta\right) \\
{\left[\ddot{\gamma}_{1}(t) \cos \theta+\ddot{\gamma}_{2}(t) \sin \theta+\dot{\theta}(t)\left(-\dot{\gamma}_{1} \sin \theta+\dot{\gamma}_{2} \cos \theta\right)\right]+\dot{\theta}(t) \theta(t)}
\end{array}\right. \\
\cdot\left[\left(\dot{\gamma}_{1}(t) \cos \theta+\dot{\gamma}_{2}(t) \sin \theta\right)^{2}+(\dot{\theta}(t))^{2}\right]^{-3}
\end{array}\right\} .
$$

Proof. By (4), we have

$$
\begin{aligned}
\dot{\gamma}(t) & =\dot{\gamma}(t) \frac{\partial}{\partial x}+\dot{\gamma}_{2}(t) \frac{\partial}{\partial y}+\dot{\theta}(t) \frac{\partial}{\partial \theta} \\
& =\dot{\gamma}_{1}(t)\left(\cos \theta X_{1}+\sin \theta X_{3}\right)+\dot{\gamma}_{2}(t)\left(\sin \theta X_{1}-\cos \theta X_{3}\right)+\dot{\theta}(t) X_{2} \\
& =\left(\dot{\gamma}_{1}(t) \cos \theta+\dot{\gamma}_{2}(t) \sin \theta\right) X_{1}+\dot{\theta}(t) X_{2}+\left(\dot{\gamma}_{1}(t) \sin \theta-\dot{\gamma}_{2}(t) \cos \theta\right) X_{3} \\
& =\left(\dot{\gamma}_{1}(t) \cos \theta+\dot{\gamma}_{2}(t) \sin \theta\right) X_{1}+\dot{\theta}(t) X_{2}+\omega\left(\dot{\gamma}_{1}(t)\right) X_{3} .
\end{aligned}
$$

By Proposition 1 and (25), we have

$$
\begin{aligned}
\nabla_{\dot{\gamma}}^{L} X_{1} & =\left(\dot{\gamma}_{1}(t) \cos \theta+\dot{\gamma}_{2}(t) \sin \theta\right) \nabla_{X_{1}}^{L} X_{1}+\dot{\theta}(t) \nabla_{X_{2}}^{L} X_{1}+\omega(\dot{\gamma}(t)) \nabla_{X_{3}}^{L} X_{1} \\
& =\dot{\theta}(t) \frac{-(L+1)}{2 L} X_{3}+\omega(\dot{\gamma}(t)) \frac{-L+1}{2} X_{2}, \\
\nabla_{\dot{\gamma}}^{L} X_{2} & =\left(\dot{\gamma}_{1}(t) \cos \theta+\dot{\gamma}_{2}(t) \sin \theta\right) \nabla_{X_{1}}^{L} X_{2}+\dot{\theta}(t) \nabla_{X_{2}}^{L} X_{2}+\omega(\dot{\gamma}(t)) \nabla_{X_{3}}^{L} X_{2} \\
& =\left(\dot{\gamma}_{1}(t) \cos \theta+\dot{\gamma}_{2}(t) \sin \theta\right) \frac{L-1}{2 L} X_{3}+\omega(\dot{\gamma}(t)) \frac{-1+L}{2} X_{1}, \\
\nabla_{\dot{\gamma}}^{L} X_{3} & =\left(\dot{\gamma}_{1}(t) \cos \theta+\dot{\gamma}_{2}(t) \sin \theta\right) \nabla_{X_{1}}^{L} X_{3}+\dot{\theta}(t) \nabla_{X_{2}}^{L} X_{3}+\omega(\dot{\gamma}(t)) \nabla_{X_{3}}^{L} X_{3} \\
& =\left(\dot{\gamma}_{1}(t) \cos \theta+\dot{\gamma}_{2}(t) \sin \theta\right) \frac{1-L}{2} X_{2}+\dot{\theta}(t) \frac{1+L}{2} X_{1} .
\end{aligned}
$$


By (25) and (26), we have

$$
\begin{aligned}
\nabla_{\dot{\gamma}}^{L} \dot{\gamma}= & \nabla_{\dot{\gamma}}^{L}\left(\dot{\gamma}_{1}(t) \cos \theta+\dot{\gamma}_{2}(t) \sin \theta\right) X_{2}+\dot{\theta}(t) X_{2}+\omega(\dot{\gamma}(t)) X_{3} \\
= & \dot{\gamma}\left(\dot{\gamma}_{1}(t) \cos \theta+\dot{\gamma}_{2}(t) \sin \theta\right) X_{1}+\left(\dot{\gamma}_{1}(t) \cos \theta+\dot{\gamma}_{2}(t) \sin \theta\right) \nabla_{\dot{\gamma}}^{L} X_{1}+\dot{\gamma}(\dot{\theta}(t)) X_{2} \\
& +\dot{\theta}(t) \nabla_{\dot{\gamma}}^{L} X_{2}+\dot{\gamma}(\omega(\dot{\gamma}(t))) X_{3}+\omega(\dot{\gamma}(t)) \nabla_{\dot{\gamma}}^{L} X_{3} \\
= & {\left[\ddot{\gamma}_{1}(t) \cos \theta+\ddot{\gamma}_{2}(t) \sin \theta+\dot{\theta}(t)\left(-\dot{\gamma}_{1} \sin \theta+\dot{\gamma}_{2} \cos \theta+L \omega(\dot{\gamma}(t))\right)\right] X_{1} } \\
& +\left[\left(\dot{\gamma}_{1}(t) \cos \theta+\dot{\gamma}_{2}(t) \sin \theta\right) \omega(\dot{\gamma}(t))(1-L)+\ddot{\theta}(t)\right] X_{2}+ \\
& \cdot\left[\left(\dot{\gamma}_{1}(t) \cos \theta+\dot{\gamma}_{2}(t) \sin \theta\right) \dot{\theta}(t) \frac{-1}{L}+\frac{\mathrm{d}}{\mathrm{d} t} \omega(\dot{\gamma}(t))\right] X_{3} .
\end{aligned}
$$

By (22) and (25), we get

$$
\begin{aligned}
&\left\|\nabla_{\dot{\gamma}}{ }^{L} \dot{\gamma}\right\|_{L}^{2}= {\left[\left(\ddot{\gamma}_{1}(t) \cos \theta+\ddot{\gamma}_{2}(t) \sin \theta+\dot{\theta}(t)\left(-\dot{\gamma}_{1}(t) \sin \theta+\dot{\gamma}_{2}(t) \cos \theta+L \omega(\dot{\gamma}(t))\right)\right]^{2}\right.} \\
&+\left[\left(\dot{\gamma}_{1}(t) \cos \theta+\dot{\gamma}_{2}(t) \sin \theta\right) \omega(\dot{\gamma}(t))(1-L)+\ddot{\theta}(t)\right]^{2} \\
&+L\left[\left(\dot{\gamma}_{1}(t) \cos \theta+\dot{\gamma}_{2}(t) \sin \theta\right) \dot{\theta}(t)\left(\frac{-1}{L}\right)+\frac{\mathrm{d}}{\mathrm{d} t}(\omega(\dot{\gamma}(t)))\right]^{2}, \\
&\|\dot{\gamma}\|_{L}^{4}=\left[\left(\dot{\gamma}_{1}(t) \cos \theta+\dot{\gamma}_{2}(t) \sin \theta\right)^{2}+(\dot{\theta}(t))^{2}+L(\omega(\dot{\gamma}(t)))^{2}\right]^{2}, \\
&\left\langle\nabla_{\dot{\gamma}} \dot{\gamma}_{\hat{\gamma}} \dot{\gamma}_{L}^{2}=\left\{\left(\dot{\gamma}_{1}(t) \cos \theta+\dot{\gamma}_{2}(t) \sin \theta\right)\right.\right. \\
&=\left[\ddot{\gamma}_{1}(t) \cos \theta+\ddot{\gamma}_{2}(t) \sin \theta+\dot{\theta}(t)\left(-\dot{\gamma}_{1} \sin \theta+\dot{\gamma}_{2} \cos \theta+L \omega(\dot{\gamma}(t))\right)\right] \\
&=+\dot{\theta}(t)\left[\left(\dot{\gamma}_{1}(t) \cos \theta+\dot{\gamma}_{2}(t) \sin \theta\right) \omega(\dot{\gamma}(t))(1-L)+\ddot{\theta}(t)\right] \\
&\left.=+L \omega(\dot{\gamma}(t))\left[\left(\dot{\gamma}_{1}(t) \cos \theta+\dot{\gamma}_{2}(t) \sin \theta\right) \dot{\theta}(t) \frac{-1}{L}+\frac{\mathrm{d}}{\mathrm{d} t}(\omega(\dot{\gamma}(t)))\right]\right\}^{2}, \\
&\|\dot{\gamma}\|_{L}^{6}=\left[\left(\dot{\gamma}_{1}(t) \cos \theta+\dot{\gamma}_{2}(t) \sin \theta\right)^{2}+(\dot{\theta}(t))^{2}+L(\omega(\dot{\gamma}(t)))^{2}\right]^{3} .
\end{aligned}
$$

By the definition of $\kappa_{\gamma}^{L}$, we get Proposition 3.

Definition 3. Let $\gamma:[a, b] \longrightarrow\left(\mathscr{R T}, g_{L}\right)$ be a Euclidean $C^{2}$-smooth regular curve in the Riemannian manifold $\left(\mathscr{R} \mathscr{T}, g_{L}\right)$; we define the intrinsic curvature $\kappa_{\gamma}^{\infty}$ of $\gamma$ at $\gamma(t)$ to be

$$
\kappa_{\gamma}^{\infty}:=\lim _{L \longrightarrow \infty} \kappa_{\gamma}^{L}
$$

if the limit exists.
We introduce the following notation: for continuous functions $f_{1}, f_{2}:(0,+\infty) \longrightarrow \mathbb{R}$,

$$
f_{1}(L) \sim f_{2}(L) \text {, as } L \longrightarrow+\infty \Leftrightarrow \lim _{L \longrightarrow \infty} \frac{f_{1}(L)}{f_{2}(L)}=1 .
$$

Proposition 4. Let $\gamma:[a, b] \longrightarrow\left(\mathscr{R T}, g_{L}\right)$ be a Euclidean $C^{2}$-smooth regular curve in the Riemannian manifold $\left(\mathscr{R} \mathscr{T}, g_{L}\right)$. Then, 


$$
\begin{aligned}
\kappa_{\gamma}^{\infty}= & \frac{\dot{\theta}^{2}+\left(\dot{\gamma}_{1} \cos \theta+\dot{\gamma}_{2} \sin \theta\right)^{2}}{|\omega(\dot{\gamma}(t))|} \text { if } \omega(\dot{\gamma}(t)) \neq 0 . \\
\kappa_{\gamma}^{\infty}= & \left\{\left\{\left[\ddot{\gamma}_{1} \cos \theta+\ddot{\gamma}_{2} \sin \theta+\dot{\theta}\left(-\dot{\gamma}_{1} \sin \theta+\dot{\gamma}_{2} \cos \theta\right)\right]^{2}+\ddot{\theta}^{2}\right\} \cdot\left[\left(\dot{\gamma}_{1} \cos \theta+\dot{\gamma}_{2} \sin \theta\right)^{2}+\dot{\theta}^{2}\right]^{-2}\right. \\
& -\left\{\left(\dot{\gamma}_{1} \cos \theta+\dot{\gamma}_{2} \sin \theta\right)\left[\ddot{\gamma}_{1} \cos \theta+\ddot{\gamma}_{2} \sin \theta+\dot{\theta}\left(-\dot{\gamma}_{1} \sin \theta+\dot{\gamma}_{2} \cos \theta\right)\right]+\ddot{\theta}_{\theta}\right\}^{2} \\
& \left.\cdot\left[\left(\dot{\gamma}_{1} \cos \theta+\dot{\gamma}_{2} \sin \theta\right)^{2}+\dot{\theta}^{2}\right]^{-3}\right\}^{(1 / 2)} \quad \text { if } \omega(\dot{\gamma}(t))=0 \text { and } \frac{\mathrm{d}}{\mathrm{d} t}(\omega(\dot{\gamma}(t)))=0, \\
\lim _{L \longrightarrow \infty} \frac{\kappa_{\gamma}^{L}}{\sqrt{L}=} & \frac{|(\mathrm{d} / \mathrm{d} t)(\omega(\dot{\gamma}(t)))|}{\left(\dot{\gamma}_{1} \cos \theta+\dot{\gamma}_{2} \sin \theta\right)^{2}+\dot{\theta}^{2}} \quad \text { if } \omega(\dot{\gamma}(t))=0 \text { and } \frac{\mathrm{d}}{\mathrm{d} t}(\omega(\dot{\gamma}(t))) \neq 0 .
\end{aligned}
$$

Proof. When $\omega(\dot{\gamma}(t)) \neq 0$, we have

$$
\begin{aligned}
\left\|\nabla_{\dot{\gamma}}^{L} \gamma\right\|_{L}^{2} & \sim \omega(\dot{\gamma}(t))^{2}\left(\dot{\theta}^{2}+\dot{\gamma}_{1}^{2} \cos ^{2} \theta+\dot{\gamma}_{2}^{2} \sin ^{2} \theta+2 \dot{\gamma}_{1} \dot{\gamma}_{2} \cos \theta \sin \theta\right) L^{2} \\
& \sim \omega(\dot{\gamma}(t))^{2}\left[\dot{\theta}^{2}+\left(\dot{\gamma}_{1} \cos \theta+\dot{\gamma}_{2} \sin \theta\right)^{2}\right] L^{2} \text { as } L \longrightarrow+\infty \\
\|\dot{\gamma}\|_{L}^{2} & \sim L \omega(\dot{\gamma}(t))^{2} \\
\left\langle\nabla_{\dot{\gamma}}^{L} \dot{\gamma}, \dot{\gamma}\right\rangle_{L}^{2} & \sim O\left(L^{2}\right) \text { as } L \longrightarrow+\infty
\end{aligned}
$$

Therefore,

$$
\begin{gathered}
\frac{\left\|\nabla_{\dot{\gamma}}^{L} \dot{\gamma}\right\|_{L}^{2}}{\|\dot{\gamma}\|_{L}^{4}} \longrightarrow \frac{\dot{\theta}^{2}+\left(\dot{\gamma}_{1} \cos \theta+\dot{\gamma}_{2} \sin \theta\right)^{2}}{\omega(\dot{\gamma}(t))^{2}} \text { as } L \longrightarrow+\infty, \\
\frac{\left\langle\nabla_{\dot{\gamma}}^{L} \dot{\gamma}, \dot{\gamma}\right\rangle_{L}^{2}}{\|\dot{\gamma}\|_{L}^{6}} \longrightarrow 0 \text { as } L \longrightarrow+\infty .
\end{gathered}
$$

If $\omega(\dot{\gamma}(t)) \neq 0$, by $(22)$, we have

$$
\kappa_{\gamma}^{\infty}=\frac{\dot{\theta}^{2}+\left(\dot{\gamma}_{1} \cos \theta+\dot{\gamma}_{2} \sin \theta\right)^{2}}{|\omega(\dot{\gamma}(t))|} .
$$

By $(24)$ and $(\mathrm{d} / \mathrm{d} t)(\omega(\dot{\gamma}(t)))=0$, we have

$$
\begin{aligned}
\kappa_{\gamma}^{\infty}= & \left\{\left\{\left[\ddot{\gamma}_{1} \cos \theta+\ddot{\gamma}_{2} \sin \theta+\dot{\theta}\left(-\dot{\gamma}_{1} \sin \theta+\dot{\gamma}_{2} \cos \theta\right)\right]^{2}+\ddot{\theta}^{2}\right\} \cdot\left[\left(\dot{\gamma}_{1} \cos \theta+\dot{\gamma}_{2} \sin \theta\right)^{2}+\dot{\theta}^{2}\right]^{-2}\right. \\
& -\left\{\left(\dot{\gamma}_{1} \cos \theta+\dot{\gamma}_{2} \sin \theta\right)\left[\ddot{\gamma}_{1} \cos \theta+\ddot{\gamma}_{2} \sin \theta+\dot{\theta}\left(-\dot{\gamma}_{1} \sin \theta+\dot{\gamma}_{2} \cos \theta\right)\right]+\ddot{\theta} \ddot{\theta}\right\}^{2} \\
& \left.\cdot\left[\left(\dot{\gamma}_{1} \cos \theta+\dot{\gamma}_{2} \sin \theta\right)^{2}+\dot{\theta}^{2}\right]^{-3}\right\}
\end{aligned}
$$

When $\omega(\dot{\gamma}(t))=0$ and $(\mathrm{d} / \mathrm{d} t)(\omega(\dot{\gamma}(t))) \neq 0$, we have

$$
\begin{aligned}
\left\|\nabla_{\dot{\gamma}}^{L} \gamma\right\|_{L}^{2} & \sim L\left[\frac{\mathrm{d}}{\mathrm{d} t}(\omega(\dot{\gamma}(t)))\right]^{2} \text { as } L \longrightarrow+\infty, \\
\|\dot{\gamma}\|_{L}^{2} & =\left(\dot{\gamma}_{1} \cos \theta+\dot{\gamma}_{2} \sin \theta\right)^{2}+\dot{\theta}^{2}, \\
\left\langle\nabla_{\dot{\gamma}}^{L} \dot{\gamma}, \dot{\gamma}\right\rangle_{L}^{2} & \sim O(1) \text { as } L \longrightarrow+\infty .
\end{aligned}
$$

If $\omega(\dot{\gamma}(t))=0$ and $(\mathrm{d} / \mathrm{d} t)(\omega(\dot{\gamma}(t)))=0$, by $(22)$, we get

$$
\lim _{L \longrightarrow \infty} \frac{\kappa_{\gamma}^{L}}{\sqrt{L}}=\frac{|(\mathrm{d} / \mathrm{d} t)(\omega(\dot{\gamma}(t)))|}{\left(\dot{\gamma}_{1} \cos \theta+\dot{\gamma}_{2} \sin \theta\right)^{2}+\dot{\theta}^{2}} .
$$

\section{The Sub-Riemannian Limit of the Geodesic Curvature of Curves on Surfaces in the Rototranslation Group}

In this section, we will compute the sub-Riemannian limit of the geodesic curvature of curves on surfaces in the 
rototranslation group. We will say that a surface $\Sigma \subset\left(\mathscr{R T}, g_{L}\right)$ is regular if $\Sigma$ is a Euclidean $C^{2}$-smooth compact and oriented surface. In particular, we will assume that there exists a Euclidean $C^{2}$-smooth function $u: \mathscr{R} \mathscr{T} \longrightarrow \mathbb{R}$ such that

$$
\Sigma=\left\{\left(x_{1}, x_{2}, \theta\right) \in \mathscr{R} \mathscr{T}: u\left(x_{1}, x_{2}, \theta\right)=0\right\},
$$

and $\quad u_{x_{1}} \partial_{x_{1}}+u_{x_{2}} \partial_{x_{2}}+u_{\theta} \partial_{\theta} \neq 0$. $\nabla_{H} u=X_{1}(u) X_{1}+X_{2}(u) X_{2}$. A point $x \in \Sigma$ is called a characteristic if $\nabla_{H} u(x)=0$. We define the characteristic set

$$
C(\Sigma):=\left\{\left(x_{1}, x_{2}, \theta\right) \in \Sigma \mid \nabla_{H} u\left(x_{1}, x_{2}, \theta\right)=0\right\} .
$$

Our computations will be local and away from characteristic points of $\Sigma$. Let us first define $p:=X_{1} u, q:=X_{2} u$, and $r:=\widetilde{X_{3}} u$. We then define

$$
\begin{aligned}
l & :=\sqrt{p^{2}+q^{2}}, \\
l_{L} & :=\sqrt{p^{2}+q^{2}+r^{2}}, \\
\bar{p} & :=\frac{p}{l}, \\
\bar{q} & :=\frac{q}{l}, \\
\overline{p_{L}} & :=\frac{p}{l_{L}} \\
\overline{q_{L}} & :=\frac{q}{l_{L}}, \\
\overline{r_{L}} & :=\frac{r}{l_{L}} .
\end{aligned}
$$

Let

In particular, $\bar{p}^{2}+\bar{q}^{2}=1$. These functions are well defined at every noncharacteristic point. Let

$$
\begin{aligned}
& v_{L}=\overline{p_{L}} X_{1}+\overline{q_{L}} X_{2}+\overline{r_{L}} \widetilde{X_{3}}, \\
& e_{1}=\bar{q} X_{1}-\bar{p} X_{2}, \\
& e_{2}=\overline{r_{L}} \bar{p} X_{1}+\overline{r_{L}} \bar{q} X_{2}-\frac{l}{l_{L}} \widetilde{X_{3}} .
\end{aligned}
$$

Then, $v_{L}$ is the Riemannian unit normal vector to $\Sigma$, and $e_{1}$ and $e_{2}$ are the orthonormal basis of $\Sigma$. On $T \Sigma$, we define a linear transformation $J_{L}: T \Sigma \longrightarrow T \Sigma$ such that

$$
J_{L}\left(e_{1}\right):=e_{2}, J_{L}\left(e_{2}\right):=-e_{1}
$$

For every $U, V \in T \Sigma$, we define $\nabla_{U}^{\Sigma, L} V=\pi \nabla_{U}^{L} V$, where $\pi: T G \longrightarrow T \Sigma$ is the projection. Then, $\nabla^{\Sigma, L}$ is the Levi-Civita connection on $\Sigma$ with respect to the metric $g_{L}$. By (27), (42), and

$$
\nabla_{\dot{\gamma}}^{\Sigma, L} \dot{\gamma}=\left\langle\nabla_{\dot{\gamma}}^{L} \dot{\gamma}, e_{1}\right\rangle_{L} e_{1}+\left\langle\nabla_{\dot{\gamma}}^{L} \dot{\gamma}, e_{2}\right\rangle_{L} e_{2}
$$

we have

$$
\begin{aligned}
& \nabla_{\dot{\gamma}}^{\Sigma, L} \dot{\gamma}=\left\{\bar{q}\left[\ddot{\gamma}_{1} \cos \theta+\ddot{\gamma}_{2} \sin \theta+\dot{\theta}\left(-\dot{\gamma}_{1} \sin \theta+\dot{\gamma}_{2} \cos \theta+L \omega(\dot{\gamma}(t))\right)\right]\right. \\
& \left.-\bar{p}\left[\left(\dot{\gamma}_{1} \cos \theta+\dot{\gamma}_{2} \sin \theta\right) \omega(\dot{\gamma}(t)(1-L)+\ddot{\theta}(t))\right]\right\} e_{1} \\
& +\left\{\begin{array}{c}
\overline{r_{L}} \bar{p}\left[\ddot{\gamma}_{1} \cos \theta+\ddot{\gamma}_{2} \sin \theta+\dot{\theta}-\dot{\gamma}_{1} \sin \theta+\dot{\gamma}_{2} \cos \theta+L \omega(\dot{\gamma}(t))\right] \\
+\overline{r_{L}} \bar{q}\left[\left(\dot{\gamma}_{1} \cos \theta+\dot{\gamma}_{2} \sin \theta\right) \omega(\dot{\gamma}(t))(1-L)+\ddot{\theta}(t)\right]-\frac{l}{l_{L}} L^{(1 / 2)}\left[\left(\dot{\gamma}_{1} \cos \theta+\dot{\gamma}_{2} \sin \theta\right) \dot{\theta} \frac{(-1)}{L}+\frac{\mathrm{d}}{\mathrm{d} t}(\omega(\dot{\gamma}(t)))\right]
\end{array}\right\}
\end{aligned}
$$

Moreover, if $\omega(\dot{\gamma}(t))=0$, then

$$
\begin{aligned}
\nabla_{\dot{\gamma}}^{\Sigma, L} \dot{\gamma}= & \left\{\bar{q}\left[\ddot{\gamma}_{1} \cos \theta+\ddot{\gamma}_{2} \sin \theta+\dot{\theta}\left(-\dot{\gamma}_{1} \sin \theta+\dot{\gamma}_{2} \cos \theta\right)\right]-\bar{p} \ddot{\theta}\right\} e_{1} \\
& +\left\{\overline{r_{L}} \bar{p}\left[\ddot{\gamma}_{1} \cos \theta+\ddot{\gamma}_{2} \sin \theta+\dot{\theta}\left(-\dot{\gamma}_{1} \sin \theta+\dot{\gamma}_{2} \cos \theta\right)\right]+\overline{r_{L}} \bar{q} \theta-\frac{l}{l_{L}} L^{(1 / 2)}\left[\left(\dot{\gamma}_{1} \cos \theta+\dot{\gamma}_{2} \sin \theta\right) \dot{\theta} \frac{(-1)}{L}+\frac{\mathrm{d}}{\mathrm{d} t}(\omega(\dot{\gamma}(t)))\right]\right\} e_{2} .
\end{aligned}
$$


Definition 4. Let $\Sigma \subset\left(\mathscr{R T}, g_{L}\right)$ be a regular surface and $\gamma:[a, b] \longrightarrow \Sigma$ be a Euclidean $C^{2}$-smooth regular curve. The geodesic curvature $\kappa_{\gamma, \Sigma}^{L}$ of $\gamma$ at $\gamma(t)$ is defined as

$$
\kappa_{\gamma, \Sigma}^{L}:=\sqrt{\frac{\left\|\nabla_{\dot{\gamma}}^{\Sigma, L} \dot{\gamma}\right\|_{\Sigma, L}^{2}}{\|\dot{\gamma}\|_{\Sigma, L}^{4}}-\frac{\left\langle\nabla_{\dot{\gamma}}^{\Sigma, L} \dot{\gamma}, \dot{\gamma}\right\rangle_{\Sigma, L}^{2}}{\|\dot{\gamma}\|_{\Sigma, L}^{6}}} .
$$

Definition 5. Let $\Sigma \subset\left(\mathscr{R T}, g_{L}\right)$ be a regular surface and $\gamma:[a, b] \longrightarrow \Sigma$ be a Euclidean $C^{2}$-smooth regular curve. We define the intrinsic geodesic curvature $\kappa_{\gamma, \Sigma}^{\infty}$ of $\gamma$ at $\gamma(t)$ to be

$$
\kappa_{\gamma, \Sigma}^{\infty}:=\lim _{L \longrightarrow+\infty} \kappa_{\gamma, \Sigma}^{L}
$$

if the limit exists.

Proposition 5. Let $\Sigma \subset\left(\mathscr{R T}, g_{L}\right)$ be a regular surface and $\gamma:[a, b] \longrightarrow \Sigma$ be a Euclidean $C^{2}$-smooth regular curve. Then,

$$
\begin{aligned}
& \kappa_{\gamma, \Sigma}^{\infty}=\frac{\left|\bar{q} \dot{\theta}+\bar{p}\left(\dot{\gamma}_{1} \cos \theta+\dot{\gamma}_{2} \sin \theta\right)\right|}{|\omega(\dot{\gamma}(t))|} \text { if } \omega(\dot{\gamma}(t)) \neq 0, \\
& \kappa_{\gamma, \Sigma}^{\infty}=0 \quad \text { if } \omega(\dot{\gamma}(t))=0 \text { and } \frac{\mathrm{d}}{\mathrm{d} t}(\omega(\dot{\gamma}(t)))=0, \\
& \lim _{L \longrightarrow+\infty} \frac{\kappa_{\gamma, \Sigma}^{L}}{\sqrt{L}}=\frac{|(\mathrm{d} / \mathrm{d} t)(\omega(\dot{\gamma}(t)))|}{\left[-\bar{p} \dot{\theta}+\bar{q}\left(\dot{\gamma}_{1} \cos \theta+\dot{\gamma}_{2} \sin \theta\right)\right]^{2}} \\
& \text { if } \omega(\dot{\gamma}(t))=0 \text { and } \frac{\mathrm{d}}{\mathrm{d} t}(\omega(\dot{\gamma}(t))) \neq 0 .
\end{aligned}
$$

Proof. By (25) and $\dot{\gamma} \in T \Sigma$, we have

$$
\dot{\gamma}(t)=\left(\dot{\gamma}_{1} \cos \theta+\dot{\gamma}_{2} \sin \theta\right) X_{1}+\dot{\theta}(t) X_{2}+\omega(\dot{\gamma}(t)) X_{3} .
$$

By (46), we have

$$
\begin{aligned}
\dot{\gamma}(t) & =a e_{1}+b e_{2}=a\left(\bar{q} X_{1}-\bar{p} X_{2}\right)+b\left(\overline{r_{L}} \bar{p} X_{1}+\overline{r_{L}} \bar{q} X_{2}-\frac{l}{l_{L}} \widetilde{X_{3}}\right) \\
& =\left(a \bar{q}+b \overline{r_{L}} \bar{p}\right) X_{1}+\left(-a \bar{p}+b \overline{r_{L}} \bar{q}\right) X_{2}-\frac{b l}{l_{L}} L^{-(1 / 2)} X_{3} .
\end{aligned}
$$

By the aforementioned first equation and the second equation, we get

$$
\left\{\begin{array}{l}
a \bar{q}+b \overline{r_{L}} \bar{p}=\dot{\gamma}_{1} \cos \theta+\dot{\gamma}_{2} \sin \theta, \\
-a \bar{p}+b \overline{r_{L}} \bar{q}=\dot{\theta}(t), \\
-\frac{b l}{l_{L}} L-\frac{1}{2}=\omega(\dot{\gamma}(t)) .
\end{array}\right.
$$

Solving the above equations, we get

$$
\left\{\begin{array}{l}
a=\left(\dot{\gamma}_{1} \cos \theta+\dot{\gamma}_{2} \sin \theta\right) \bar{q}-\bar{p} \dot{\theta}(t), \\
b=-L \frac{1}{2} \frac{l_{L}}{l} \omega(\dot{\gamma}(t)) .
\end{array}\right.
$$

Then, we get

$\dot{\gamma}=\left[-\bar{p} \dot{\theta}(t)+\bar{q}\left(\dot{\gamma}_{1} \cos \theta+\dot{\gamma}_{2} \sin \theta\right)\right] e_{1}-\frac{l_{L}}{l} L^{(1 / 2)} \omega(\dot{\gamma}(t)) e_{2}$.

By (26), we have

$$
\begin{aligned}
\left\|\nabla_{\dot{\gamma}}^{\Sigma, L} \dot{\gamma}\right\|_{\Sigma, L}^{2}= & \left\{\bar{q}\left[\ddot{\gamma}_{1} \cos \theta+\ddot{\gamma}_{2} \sin \theta+\dot{\theta}\left(-\dot{\gamma}_{1} \sin \theta+\dot{\gamma}_{2} \cos \theta+L \omega(\dot{\gamma}(t))\right)\right]-\bar{p}\left[\left(\dot{\gamma}_{1} \cos \theta+\dot{\gamma}_{2} \sin \theta\right) \omega(\dot{\gamma}(t))(1-L)+\ddot{\theta}(t)\right]\right\}^{2} \\
& +\left\{\begin{array}{c}
\bar{r}_{L} \bar{p}\left[\ddot{\gamma}_{1} \cos \theta+\ddot{\gamma}_{2} \sin \theta+\dot{\theta}\left(-\dot{\gamma}_{1} \sin \theta+\dot{\gamma}_{2} \cos \theta+L \omega(\dot{\gamma}(t))\right)\right] \\
+\overline{r_{L}} \bar{q}\left[\left(\dot{\gamma}_{1} \cos \theta+\dot{\gamma}_{2} \sin \theta\right) \omega(\dot{\gamma}(t))(1-L)+\ddot{\theta}(t)\right]-\frac{l}{l_{L}} L^{(1 / 2)}\left[\left(\dot{\gamma}_{1} \cos \theta+\dot{\gamma}_{2} \sin \theta\right) \dot{\theta} \frac{(-1)}{L}+\frac{\mathrm{d}}{\mathrm{d} t} \omega(\dot{\gamma}(t))\right]
\end{array}\right]^{2} \\
& \sim\left[\bar{q} \dot{\theta} \omega(\dot{\gamma}(t))+\bar{p}\left(\dot{\gamma}_{1} \cos \theta+\dot{\gamma}_{2} \sin \theta\right) \omega(\dot{\gamma}(t))\right]^{2} L^{2} \\
& +\left[\overline{r_{L}} \bar{p} \dot{\theta} \omega(\dot{\gamma}(t))-\overline{r_{L}} \bar{q}\left(\dot{\gamma}_{1} \cos \theta+\dot{\gamma}_{2} \sin \theta\right) \omega(\dot{\gamma}(t))\right]^{2} L^{2} \\
= & {\left[\bar{q} \dot{\theta}+\bar{p}\left(\dot{\gamma}_{1} \cos \theta+\dot{\gamma}_{2} \sin \theta\right)\right]^{2} \omega(\dot{\gamma}(t)) L^{2}+\left\{\overline{r_{L}}\left[\bar{p}-\bar{q}\left(\dot{\gamma}_{1} \cos \theta+\dot{\gamma}_{2} \sin \theta\right)\right]\right\}^{2} \omega^{2}(\dot{\gamma}(t)) L^{2} . }
\end{aligned}
$$

Similarly, we have that when $\omega(\dot{\gamma}(t)) \neq 0$, 


$$
\|\dot{\gamma}\|_{\Sigma, L}=\sqrt{\left[-\bar{p} \dot{\theta}+\bar{q}\left(\dot{\gamma}_{1} \cos \theta+\dot{\gamma}_{2} \sin \theta\right)\right]^{2}+\left(\frac{l_{L}}{l}\right)^{2} L \omega^{2}(\dot{\gamma}(t))} \sim L^{(1 / 2)}|\omega(\dot{\gamma}(t))| \text { as } L \longrightarrow+\infty .
$$

By (45) and (55), we have

$$
\begin{aligned}
\left\langle\nabla_{\dot{\gamma}}^{\Sigma, L} \dot{\gamma}, \dot{\gamma}\right\rangle_{\Sigma, L}= & {\left[-\bar{p} \dot{\theta}+\bar{q}\left(\dot{\gamma}_{1} \cos \theta+\dot{\gamma}_{2} \sin \theta\right)\right] } \\
& \left\{-\bar{p}\left[\left(\dot{\gamma}_{1} \cos \theta+\dot{\gamma}_{2} \sin \theta\right) \omega((\dot{\gamma}(t))(1-L)+\ddot{\theta}(t))\right]\right. \\
& \left.+\bar{q}\left[\ddot{\gamma_{1}} \cos \theta+\ddot{\gamma_{2}} \sin \theta+\dot{\theta}\left(-\dot{\gamma}_{1} \sin \theta+\dot{\gamma}_{2} \cos \theta+L \omega(\dot{\gamma}(t))\right)\right]\right\}-\frac{l}{l_{L}} L^{(1 / 2)}(\omega(\dot{\gamma}(t))) \\
& \left\{\overline{r_{L}} \bar{p}\left[\ddot{\gamma}_{1} \cos \theta+\ddot{\gamma}_{2} \sin \theta+\dot{\theta}\left(-\dot{\gamma}_{1} \sin \theta+\dot{\gamma}_{2} \cos \theta+L \omega(\dot{\gamma}(t))\right)\right]\right. \\
& +\overline{r_{L}} \bar{q}\left[\left(\dot{\gamma}_{1} \cos \theta+\dot{\gamma}_{2} \sin \theta\right) \omega(\dot{\gamma}(t))(1-L)+\ddot{\theta}(t)\right] \\
& \left.-\frac{l}{l_{L}} L^{(1 / 2)}\left[\left(\dot{\gamma}_{1} \cos \theta+\dot{\gamma}_{2} \sin \theta\right) \dot{\theta} \frac{(-1)}{L}+\frac{\mathrm{d}}{\mathrm{d} t}(\omega(\dot{\gamma}(t)))\right]\right\} \sim M_{0} L
\end{aligned}
$$

where $M_{0}$ does not depend on $L$. By (47), we have

$$
\begin{aligned}
k_{\gamma, \Sigma}^{\infty} & =\lim _{L \longrightarrow+\infty} k_{\gamma, \Sigma}^{L}=\lim _{L \longrightarrow+\infty} \sqrt{\frac{\left[\bar{q} \dot{\theta}+\bar{p}\left(\dot{\gamma}_{1} \cos \theta+\dot{\gamma}_{2} \sin \theta\right)\right]^{2}+{\overline{r_{L}}}^{2}\left[\bar{p}-\bar{q}\left(\dot{\gamma}_{1} \cos \theta+\dot{\gamma}_{2} \sin \theta\right)\right]^{2}}{\left(\omega^{2}(\dot{\gamma}(t))\right)}} \\
& =\frac{\left|\bar{q} \theta+\bar{p}\left(\dot{\gamma}_{1} \cos \theta+\dot{\gamma}_{2} \sin \theta\right)\right|}{|\omega(\dot{\gamma}(t))|},
\end{aligned}
$$

if $\omega(\dot{\gamma}(t)) \neq 0$. When $\omega(\dot{\gamma}(t))=0$ and $(\mathrm{d} / \mathrm{d} t)(\omega(\dot{\gamma}(t)))=0$, we have 


$$
\begin{aligned}
\left\|\nabla_{\dot{\gamma}}^{\Sigma, L} \dot{\gamma}\right\|_{\Sigma, L}^{2}= & \left\{\bar{q}\left[\ddot{\gamma}_{1} \cos \theta+\ddot{\gamma}_{2} \sin \theta+\dot{\theta}\left(-\dot{\gamma}_{1} \sin \theta+\dot{\gamma}_{2} \cos \theta\right)\right]-\bar{p} \ddot{\theta}\right\}^{2} \\
& +\left\{\overline{r_{L}} \bar{p}\left[\ddot{\gamma}_{1} \cos \theta+\ddot{\gamma}_{2} \sin \theta+\dot{\theta}\left(-\dot{\gamma}_{1} \sin \theta+\dot{\gamma}_{2} \cos \theta\right)\right]\right. \\
& \left.+\overline{r_{L}} \bar{q} \ddot{\theta}(t)-\frac{l}{l_{L}} L^{(1 / 2)}\left(\dot{\gamma}_{1} \cos \theta+\dot{\gamma}_{2} \sin \theta\right) \dot{\theta} \frac{(-1)}{L}\right\}^{2} \\
& \sim\left\{\bar{q}\left[\ddot{\gamma_{1}} \cos \theta+\ddot{\gamma}_{2} \sin \theta+\dot{\theta}\left(-\dot{\gamma}_{1} \sin \theta+\dot{\gamma}_{2} \cos \theta\right)\right]-\bar{p} \ddot{\theta}\right\}^{2} \text { as } L \longrightarrow+\infty \\
\|\dot{\gamma}\|_{\Sigma, L}= & \left|-\bar{p} \dot{\theta}+\bar{q}\left(\dot{\gamma}_{1} \cos \theta+\dot{\gamma}_{2} \sin \theta\right)\right|, \\
\left\langle\nabla_{\dot{\gamma}}^{\Sigma, L} \dot{\gamma}, \dot{\gamma}\right\rangle_{\Sigma, L}= & {\left[-\bar{p} \dot{\theta}+\bar{q}\left(\dot{\gamma}_{1} \cos \theta+\dot{\gamma}_{2} \sin \theta\right)\right] } \\
& \cdot\left\{\bar{q}\left[\ddot{\gamma_{1}} \cos \theta+\ddot{\gamma}_{2} \sin \theta+\dot{\theta}\left(-\dot{\gamma}_{1} \sin \theta+\dot{\gamma}_{2} \cos \theta\right)\right]-\bar{p} \ddot{\theta}\right\}:=A B .
\end{aligned}
$$

By (60)-(62) and (47), we get

When $\omega(\dot{\gamma}(t))=0$ and $(\mathrm{d} / \mathrm{d} t)(\omega(\dot{\gamma}(t))) \neq 0$, we have

$$
\kappa_{\gamma, \Sigma}^{\infty}=\sqrt{\frac{A^{2}}{B^{4}}-\frac{A^{2} B^{2}}{B^{6}}}=0 .
$$

$$
\begin{aligned}
\left\|\nabla_{\dot{\gamma}}^{\Sigma, L} \dot{\gamma}, \dot{\gamma}\right\|_{\Sigma, L}^{2} & \sim \frac{l^{2}}{l_{L}^{2}} L\left[\frac{\mathrm{d}}{\mathrm{d} t}(\omega(\dot{\gamma}(t)))\right]^{2} \sim L\left[\frac{\mathrm{d}}{\mathrm{d} t}(\omega(\dot{\gamma}(t)))\right]^{2}, \\
\|\dot{\gamma}\|_{\Sigma, L} & =\left|-\bar{p} \dot{\theta}+\bar{q}\left(\dot{\gamma}_{1} \cos \theta+\dot{\gamma}_{2} \sin \theta\right)\right|, \\
\left\langle\nabla_{\dot{\gamma}}^{\Sigma, L} \dot{\gamma}, \dot{\gamma}\right\rangle_{\Sigma, L} & =O(1), \\
\lim _{L \longrightarrow+\infty} \frac{\kappa_{\gamma, \Sigma}^{L}}{\sqrt{L}} & =\lim _{L \longrightarrow+\infty} \frac{1}{\sqrt{L}} \sqrt{\frac{L[(\mathrm{~d} / \mathrm{d} t)(\omega(\dot{\gamma}(t)))]^{2}}{\left|-\bar{p} \dot{\theta}+\bar{q}\left(\dot{\gamma}_{1} \cos \theta+\dot{\gamma}_{2} \sin \theta\right)\right|^{4}}}=\frac{|(\mathrm{d} / \mathrm{d} t)(\omega(\dot{\gamma}(t)))|}{\left[-\bar{p} \dot{\theta}+\bar{q}\left(\dot{\gamma}_{1} \cos \theta+\dot{\gamma}_{2} \sin \theta\right)\right]^{2}},
\end{aligned}
$$

if $\omega(\dot{\gamma}(t))=0$ and $(\mathrm{d} / \mathrm{d} t)(\omega(\dot{\gamma}(t))) \neq 0$, so we get $(50)$.

Definition 6. Let $\Sigma \subset\left(\mathscr{R} \mathscr{T}, g_{L}\right)$ be a regular surface. Let $\gamma:[a, b] \longrightarrow \sum$ be a Euclidean $C^{2}$-smooth regular curve. The signed geodesic curvature $\kappa_{\gamma, \Sigma}^{L, s}$ of $\gamma$ at $\gamma(t)$ is defined as

$$
\kappa_{\gamma, \Sigma}^{L, s}:=\frac{\left\langle\nabla_{\dot{\gamma}}^{\Sigma, L} \dot{\gamma}, J_{L}(\dot{\gamma})\right\rangle_{\Sigma, L}}{\|\dot{\gamma}\|_{\Sigma, L}^{3}},
$$

where $J_{L}$ is defined by (43).

Definition 7. Let $\Sigma \subset\left(\mathscr{R} \mathscr{T}, g_{L}\right)$ be a regular surface. Let $\gamma:[a, b] \longrightarrow \Sigma$ be a Euclidean $C^{2}$-smooth regular curve. We define the intrinsic geodesic curvature $\kappa_{\gamma, \Sigma}^{\infty}$ of $\gamma$ at the noncharacteristic point $\gamma(t)$ to be

$$
\kappa_{\gamma, \Sigma}^{\infty, s}:=\lim _{L \longrightarrow+\infty} \kappa_{\gamma, \Sigma}^{L, s}
$$

if the limit exists.

Proposition 6. Let $\Sigma \subset\left(\mathscr{R} \mathscr{T}, g_{L}\right)$ be a regular surface. Let $\gamma:[a, b] \longrightarrow \Sigma$ be a Euclidean $C^{2}$-smooth regular curve. Then,

$$
\begin{aligned}
\kappa_{\gamma, \Sigma}^{\infty, s} & =\frac{\bar{q} \dot{\theta}+\bar{p}\left(\dot{\gamma}_{1} \cos \theta+\dot{\gamma}_{2} \sin \theta\right)}{|\omega(\dot{\gamma}(t))|} \quad \text { if } \omega(\dot{\gamma}(t)) \neq 0, \\
\kappa_{\gamma, \Sigma}^{\infty, s} & =0 \quad \text { if } \omega(\dot{\gamma}(t))=0 \text { and } \frac{\mathrm{d}}{\mathrm{d} t}(\omega(\dot{\gamma}(t)))=0, \\
\lim _{L \longrightarrow+\infty} \frac{k_{\gamma, \Sigma}^{L, s}}{\sqrt{L}} & =\frac{\left[\bar{p} \dot{\theta}-\bar{q}\left(\dot{\gamma}_{1} \cos \theta+\dot{\gamma}_{2} \sin \theta\right)\right](\mathrm{d} / \mathrm{d} t)(\omega(\dot{\gamma}(t)))}{\left|-\bar{p} \dot{\theta}+\bar{q}\left(\dot{\gamma}_{1} \cos \theta+\dot{\gamma}_{2} \sin \theta\right)\right|^{3}}
\end{aligned}
$$

if $\omega(\dot{\gamma}(t))=0$ and $(\mathrm{d} / \mathrm{d} t)(\omega(\dot{\gamma}(t))) \neq 0$. 
Proof. By (43) and (55), we get

$$
\begin{aligned}
J_{L}(\dot{\gamma}) & =\left[-\bar{p} \dot{\theta}+\bar{q}\left(\dot{\gamma}_{1} \cos \theta+\dot{\gamma}_{2} \sin \theta\right)\right] J_{L}\left(e_{1}\right)-\frac{l_{L}}{l} L^{(1 / 2)} \omega(\dot{\gamma}(t)) J_{L}\left(e_{2}\right) \\
& =\frac{l_{L}}{l} L^{(1 / 2)} \omega(\dot{\gamma}(t)) e_{1}+\left[-\bar{p} \dot{\theta}+\bar{q}\left(\dot{\gamma}_{1} \cos \theta+\dot{\gamma}_{2} \sin \theta\right)\right] e_{2} .
\end{aligned}
$$

By (45) and the above equation, we have

$$
\begin{aligned}
& \left\langle\nabla_{\dot{\gamma}}{ }^{\Sigma, L} \dot{\gamma}, J_{L}(\dot{\gamma})\right\rangle=\frac{l_{L}}{l} L^{(1 / 2)} \omega(\dot{\gamma}(t))\left\{\bar{q}\left[\ddot{\gamma}_{1} \cos \theta+\ddot{\gamma}_{2} \sin \theta+\dot{\theta}-\dot{\gamma}_{1} \sin \theta+\dot{\gamma}_{2} \cos \theta+L \omega(\dot{\gamma}(t))\right]\right. \\
& \left.-\bar{p}\left[\left(\dot{\gamma}_{1} \cos \theta+\dot{\gamma}_{2} \sin \theta\right) \omega(\dot{\gamma}(t))(1-L)+\ddot{\theta}(t)\right]\right\}+\left[-\bar{p} \dot{\theta}+\bar{q}\left(\dot{\gamma}_{1} \cos \theta+\dot{\gamma}_{2} \sin \theta\right)\right]
\end{aligned}
$$

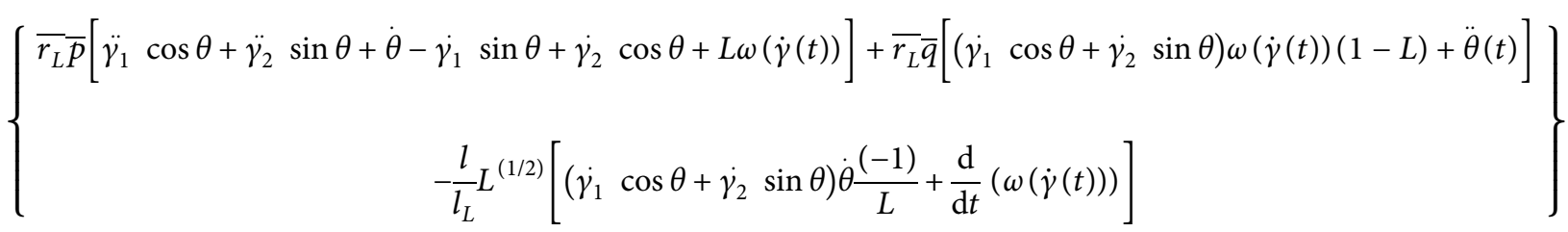

$$
\begin{aligned}
& \sim \frac{l_{L}}{l} L^{(3 / 2)}\left[\bar{q} \dot{\theta}+\bar{p}\left(\dot{\gamma}_{1} \cos \theta+\dot{\gamma}_{2} \sin \theta\right)\right] \omega^{2}(\dot{\gamma}(t)) \\
& \sim L^{(3 / 2)} \omega^{2}(\dot{\gamma}(t))\left[\bar{q} \dot{\theta}+\bar{p}\left(\dot{\gamma}_{1} \cos \theta+\dot{\gamma}_{2} \sin \theta\right)\right] \text { as } L \longrightarrow+\infty
\end{aligned}
$$

So, we get

$$
\kappa_{\gamma, \Sigma}^{L, s}=\frac{\left\langle\nabla_{\dot{\gamma}}^{\Sigma, L} \dot{\gamma}, J_{L}(\dot{\gamma})\right\rangle_{\Sigma, L}}{\|\dot{\gamma}\|_{\Sigma, L}^{3}}=\frac{L^{(3 / 2)}\left[\bar{q} \dot{\theta}+\bar{p}\left(\dot{\gamma}_{1} \cos \theta+\dot{\gamma}_{2} \sin \theta\right)\right] \omega^{2}(\dot{\gamma}(t))}{L^{(3 / 2)}|\omega(\dot{\gamma}(t))|^{3}} .
$$

Furthermore,

$$
\kappa_{\gamma, \Sigma}^{\infty, s}=\lim _{L \longrightarrow+\infty} k_{\gamma, \Sigma}^{L, s}=\frac{\bar{q} \dot{\theta}+\bar{p}\left(\dot{\gamma}_{1} \cos \theta+\dot{\gamma}_{2} \sin \theta\right)}{|\omega(\dot{\gamma}(t))|} .
$$

$$
\begin{aligned}
\left\langle\nabla_{\dot{\gamma}}^{\Sigma, L} \dot{\gamma}, J_{L}(\dot{\gamma})\right\rangle_{L, \Sigma}= & \left.-\bar{p} \dot{\theta}+\bar{q}\left(\dot{\gamma}_{1} \cos \theta+\dot{\gamma}_{2} \sin \theta\right)\right] \\
& \left\{\begin{array}{c}
\overline{r_{L}} \bar{p}\left[\ddot{\gamma_{1}} \cos \theta+\ddot{\gamma}_{2} \sin \theta+\dot{\theta}\left(-\dot{\gamma}_{1} \sin \theta+\dot{\gamma}_{2} \cos \theta\right)\right] \\
+\overline{r_{L}} \bar{q} \theta-\frac{l}{l_{L}} L^{(1 / 2)}\left(\dot{\gamma}_{1} \cos \theta+\dot{\gamma}_{2} \sin \theta\right) \dot{\theta} \frac{(-1)}{L}
\end{array}\right\} \sim M_{0} L^{-(1 / 2)} \text { as } L \longrightarrow+\infty .
\end{aligned}
$$


So, $\quad \kappa_{\gamma, \Sigma}^{\infty, s}=0 . \quad$ When $\quad \omega(\dot{\gamma}(t))=0 \quad$ and $(\mathrm{d} / \mathrm{d} t)(\omega(\dot{\gamma}(t))) \neq 0$, we have

$$
\begin{aligned}
\left\langle\nabla_{\dot{\gamma}}^{\Sigma, L} \dot{\gamma}, J_{L}(\dot{\gamma})\right\rangle_{L, \Sigma}= & {\left[-\bar{p} \dot{\theta}+\bar{q}\left(\dot{\gamma}_{1} \cos \theta+\dot{\gamma}_{2} \sin \theta\right)\right] } \\
& \cdot\left\{\begin{array}{c}
\overline{r_{L}} \bar{q}\left[\ddot{\gamma}_{1} \cos \theta+\ddot{\gamma}_{2} \sin \theta+\dot{\theta}\left(-\dot{\gamma}_{1} \sin \theta+\dot{\gamma}_{2} \cos \theta\right)\right] \\
+\overline{r_{L}} \bar{q} \ddot{\theta}-\frac{l}{l_{L}} L^{(1 / 2)}\left[\left(\dot{\gamma}_{1} \cos \theta+\dot{\gamma}_{2} \sin \theta\right)\right] \dot{\theta} \frac{(-1)}{L}+\frac{\mathrm{d}}{\mathrm{d} t}(\omega(\dot{\gamma}(t)))
\end{array}\right\} \\
& \sim\left[-\bar{p} \dot{\theta}+\bar{q}\left(\dot{\gamma}_{1} \cos \theta+\dot{\gamma}_{2} \sin \theta\right)\right]\left(-\frac{l}{l_{L}}\right) \frac{\mathrm{d}}{\mathrm{d} t}(\omega(\dot{\gamma}(t))) L^{(1 / 2)} \text { as } L \longrightarrow+\infty, \\
& \sim-\left[-\bar{p} \dot{\theta}+\bar{q}\left(\dot{\gamma}_{1} \cos \theta+\dot{\gamma}_{2} \sin \theta\right)\right] \frac{\mathrm{d}}{\mathrm{d} t}(\omega(\dot{\gamma}(t))) L^{(1 / 2)} \text { as } L \longrightarrow+\infty .
\end{aligned}
$$

We get

$$
\begin{aligned}
\kappa_{\gamma, \Sigma}^{\infty, s} & =\lim _{L \longrightarrow+\infty} \frac{\kappa_{\gamma, \Sigma}^{L}}{\sqrt{L}}=\lim _{L \longrightarrow+\infty} \frac{-\left[-\bar{p} \dot{\theta}+\bar{q}\left(\dot{\gamma}_{1} \cos \theta+\dot{\gamma}_{2} \sin \theta\right)\right](\mathrm{d} / \mathrm{d} t)(\omega(\dot{\gamma}(t))) L^{(1 / 2)}}{\left|-\bar{p} \dot{\theta}+\bar{q}\left(\dot{\gamma}_{1} \cos \theta+\dot{\gamma}_{2} \sin \theta\right)\right|^{3} \sqrt{L}} \\
& =\frac{\left[\bar{p} \dot{\theta}-\bar{q}\left(\dot{\gamma}_{1} \cos \theta+\dot{\gamma}_{2} \sin \theta\right)\right](\mathrm{d} / \mathrm{d} t)(\omega(\dot{\gamma}(t)))}{\left|-\bar{p} \dot{\theta}+\bar{q}\left(\dot{\gamma}_{1} \cos \theta+\dot{\gamma}_{2} \sin \theta\right)\right|^{3}}
\end{aligned}
$$

\section{The Sub-Riemannian Limit of the \\ Riemannian Gaussian Curvature of Surfaces in the Rototranslation Group}

In this section, we will compute the sub-Riemannian limit of the Riemannian Gaussian curvature of surfaces in the $\mathscr{R} \mathscr{T}$ group. We define the second fundamental form $I I^{L}$ of the embedding of $\Sigma$ into $\left(\mathscr{R} \mathscr{T}, g_{L}\right)$ :

$$
I I^{L}=\left(\begin{array}{cc}
\left\langle\nabla_{e_{1}}^{L} v_{L}, e_{1}\right\rangle_{L} & \left\langle\nabla_{e_{1}}^{L} v_{L}, e_{2}\right\rangle_{L} \\
\left\langle\nabla_{e_{2}}^{L} v_{L}, e_{1}\right\rangle_{L} & \left\langle\nabla_{e_{2}}^{L} v_{L}, e_{2}\right\rangle_{L}
\end{array}\right) .
$$

We have the following theorem.

Theorem 1. The second fundamental form $I I^{L}$ of the embedding of $\Sigma$ into $\left(\mathscr{R T}, g_{L}\right)$ is given by

$$
I I^{L}=\left(\begin{array}{ll}
h_{11} & h_{12} \\
h_{21} & h_{22}
\end{array}\right),
$$

where

$$
\begin{aligned}
& h_{11}=\frac{l}{l_{L}}\left(X_{1}(\bar{p})+X_{2}(\bar{q})\right)-\overline{r_{L}} \frac{\bar{p} \bar{q}}{\sqrt{L}} \\
& h_{12}=h_{21}=-\frac{l_{L}}{l}\left\langle e_{1}, \nabla_{H} \overline{r_{L}}\right\rangle_{L}-\frac{1}{2 \sqrt{L}}\left(\bar{p}_{L}^{2}-\bar{q}_{L}^{2}\right)-\frac{\sqrt{L}}{2}-\frac{\bar{r}_{L}^{2}}{2 \sqrt{L}}\left(\bar{p}^{2}-\bar{q}^{2}\right), \\
& h_{22}=-\frac{l^{2}}{l_{L}^{2}}\left\langle e_{2}, \nabla_{H}^{L}\left(\frac{r}{l}\right)\right\rangle_{L}+\widetilde{X_{3}}\left(\tilde{r_{L}}\right)+\frac{1}{\sqrt{L}} \overline{p_{L} q_{L} r_{L}}+\frac{1}{\sqrt{L}} \bar{p} \bar{q} \bar{r}_{L}^{3} .
\end{aligned}
$$


Proof. Since $\left\langle e_{1}, v_{L}\right\rangle_{L}=0$ and $\left\langle e_{2}, v_{L}\right\rangle_{L}=0$, we have

$$
\left\langle\nabla_{e_{1}}^{L} v_{L}, e_{1}\right\rangle_{L}=-\left\langle\nabla_{e_{1}}^{L} e_{1}, v_{L}\right\rangle_{L},\left\langle\nabla_{e_{2}}^{L} v_{L}, e_{2}\right\rangle_{L}=-\left\langle\nabla_{e_{2}}^{L} e_{2}, v_{L}\right\rangle_{L} .
$$

Using the definition of the connection, identities in (5), and grouping terms, we have

$$
\begin{aligned}
\nabla_{e_{1}}^{L} e_{1}= & \nabla_{\bar{q} X_{1}-\bar{p} X_{2}}^{L} \bar{q} X_{1}-\bar{p} X_{2}=\bar{q}\left(X_{1}(\bar{q}) X_{1}+\bar{q} \nabla_{X_{1}}^{L} X_{1}-X_{1}(\bar{p}) X_{2}-\bar{p} \nabla_{X_{1}}^{L} X_{2}\right) \\
& -\bar{p}\left(X_{2}(\bar{q}) X_{1}+\bar{q} \nabla_{X_{2}}^{L} X_{1}-X_{2}(\bar{p}) X_{2}-\bar{p} \nabla_{X_{2}}^{L} X_{2}\right) \\
= & \bar{q}\left(X_{1}(\bar{q}) X_{1}-X_{1}(\bar{p}) X_{2}+\bar{p} \frac{L-1}{2 L} X_{3}\right)-\bar{p}\left(X_{2}(\bar{q}) X_{1}+\bar{q}\left(\frac{-(L+1)}{2 L}\right) X_{3}-X_{2}(\bar{p}) X_{2}\right) \\
= & {\left[\bar{q} X_{1}(\bar{q})-\bar{p} X_{2}(\bar{q})\right] X_{1}-\left[\bar{q} X_{1}(\bar{p})-\bar{p} X_{2}(\bar{p})\right] X_{2}-\left(\bar{p} \bar{q} \frac{L-1}{2 L}-\bar{p} \bar{q} \frac{L+1}{2 L}\right) X_{3} } \\
= & {\left[\bar{q} X_{1}(\bar{q})-\bar{p} X_{2}(\bar{q})\right] X_{1}-\left[\bar{q} X_{1}(\bar{p})-\bar{p} X_{2}(\bar{p})\right] X_{2}+\frac{\bar{p} \bar{q}}{L} X_{3} . }
\end{aligned}
$$

Since $\bar{p}^{2}+\bar{q}^{2}=1$, we have $\bar{p} X_{i} \bar{p}+\bar{q} X_{i} \bar{q}=0, i=1,2,3$. Thus, $\bar{q} X_{1} \bar{q}=-\bar{p} X_{1} \bar{p}$ and $\bar{q} X_{2} \bar{q}=-\bar{p} X_{2} \bar{p}$, and we have

$$
\begin{aligned}
\nabla_{e_{1}}^{L} e_{1}= & -\bar{p}\left(X_{1}(\bar{p})+X_{2}(\bar{q})\right) X_{1} \\
& -\bar{q}\left(X_{1}(\bar{p})+X_{2}(\bar{q})\right) X_{2}+\frac{\bar{p} \bar{q}}{L} X_{3} .
\end{aligned}
$$
obtain

Next, we compute the inner product of this with $v_{L}$, we

$$
\begin{aligned}
h_{11} & =-\left\langle\nabla_{e_{1}}^{L} e_{1}, v_{L}\right\rangle_{L}=\overline{p p}_{L}\left(X_{1}(\bar{p})+X_{2}(\bar{q})\right)+\overline{q q}_{L}\left(X_{1}(\bar{p})+X_{2}(\bar{q})\right)-\overline{r_{L}} \frac{\bar{p} \bar{q}}{L} \sqrt{L} \\
& =\frac{p}{l} \frac{p}{l_{L}}\left(X_{1}(\bar{p})+X_{2}(\bar{q})\right)+\frac{q}{l} \frac{q}{l_{L}}\left(X_{1}(\bar{p})+X_{2}(\bar{q})\right)-\overline{r_{L}} \frac{\bar{p} \bar{q}}{\sqrt{L}} \\
& =\frac{1}{l l_{L}}\left(p^{2}+q^{2}\right) X_{1}(\bar{p})+\frac{1}{l l_{L}}\left(p^{2}+q^{2}\right) X_{2}(\bar{q})-\overline{r_{L}} \frac{\bar{p} \bar{q}}{\sqrt{L}}=\frac{l}{l_{L}}\left(X_{1}(\bar{p})+X_{2}(\bar{q})\right)-\overline{r_{L}} \frac{\bar{p} \bar{q}}{\sqrt{L}}
\end{aligned}
$$

To compute $h_{12}$ and $h_{21}$, using the definition of the connection, we obtain

$$
\begin{aligned}
\nabla_{e_{1}}^{L} e_{2}= & \nabla_{\bar{q} X_{1}-\bar{p} X_{2}}^{L} \overline{r_{L}} \bar{p} X_{1}+\overline{r_{L}} \bar{q} X_{2}-\frac{l}{l_{L}} L^{-(1 / 2)} X_{3}=\bar{q} \nabla_{X_{1}}^{L} \overline{r_{L}} \bar{p} X_{1}+\overline{r_{L}} \bar{q} X_{2}-\frac{l}{l_{L}} L^{-(1 / 2)} X_{3} \\
& -\bar{p} \nabla_{X_{2}}^{L} \overline{r_{L}} \bar{p} X_{1}+\overline{r_{L}} \bar{q} X_{2}-\frac{l}{l_{L}} L-\frac{1}{2} X_{3} \\
= & \bar{q}\left(X_{1}\left(\overline{r_{L}} \bar{p}\right) X_{1}+\overline{r_{L}} \bar{p} \nabla_{X_{1}}^{L} X_{1}+X_{1}\left(\overline{r_{L}} \bar{p}\right) X_{2}+\overline{r_{L}} \bar{p} \nabla_{X_{1}}^{L} X_{2}-X_{1}\left(\frac{l}{l_{L}}\right) L^{-(1 / 2)} X_{3}-\frac{l}{l_{L}} L^{-(1 / 2)} \nabla_{X_{1}}^{L} X_{3}\right) \\
& -\bar{p}\left(X_{2}\left(\overline{r_{L}} \bar{p}\right) X_{1}+\overline{r_{L}} \bar{p} \nabla_{X_{2}}^{L} X_{1}+X_{2}\left(\overline{r_{L}} \bar{p}\right) X_{2}+\overline{r_{L}} \bar{p} \nabla_{X_{2}}^{L} X_{2}-X_{2}\left(\frac{l}{l_{L}}\right) L^{-(1 / 2)} X_{3}-\frac{l}{l_{L}} L^{-(1 / 2)} \nabla_{X_{2}}^{L} X_{3}\right) \\
= & \left(\bar{q} X_{1}\left(\overline{r_{L}} \bar{p}\right)-\bar{p} X_{2}\left(\overline{r_{L}} \bar{p}\right)+\frac{\bar{p} l}{l_{L} \sqrt{L}} \frac{1+L}{2}\right) X_{1}+\left(\bar{q} X_{1}\left(\overline{r_{L}} \bar{q}\right)-\frac{\bar{q} l}{l_{L} \sqrt{L}} \frac{1-L}{2}-\bar{p} X_{2}\left(\overline{r_{L}} \bar{q}\right)\right) X_{2} \\
& +\left(-\frac{\bar{q}}{\sqrt{L}} X_{1}\left(\frac{l}{l_{L}}\right)+\frac{\bar{p}}{\sqrt{L}} X_{2}\left(\frac{l}{l_{L}}\right)+\frac{\overline{r_{L}}}{2}\left[1+\frac{1}{L}\left(\bar{p}^{2}-\bar{q}^{2}\right)\right]\right) X_{3} .
\end{aligned}
$$


Next, we compute the inner product of this with $v_{L}$. Using the product rule and the identity $\overline{q_{L}} \bar{p}=\overline{p_{L}} \bar{q}$, we obtain

$$
\begin{aligned}
\left\langle\nabla_{e_{1}} e_{2}, v_{L}\right\rangle_{L}= & \left(\overline{p_{L}} \bar{q} \bar{p}+{\overline{q_{L}}}^{2}\right) X_{1} \overline{r_{L}}-\left({\overline{p_{L}}}^{2}+\overline{q_{L}} \bar{p} \bar{q}\right) X_{2} \overline{r_{L}}+\overline{p_{L} r_{L}} \bar{q} X_{1} \bar{p}+\overline{r_{L} q_{L}} \bar{q} X_{1} \bar{q} \\
& -\overline{r_{L}} \bar{p}\left(\overline{p_{L}} X_{2} \bar{p}+\overline{q_{L}} X_{2} \bar{q}\right)+\frac{1}{2 \sqrt{L}}\left(\bar{p}_{L}^{2}-\bar{q}_{L}^{2}\right)+\frac{\sqrt{L}}{2}+\frac{\bar{r}_{L}^{2}}{2 \sqrt{L}}\left(\bar{p}^{2}-\bar{q}^{2}\right)+\overline{r_{L}} \bar{p} X_{2}\left(\frac{l}{l_{L}}\right)-\overline{r_{L}} \bar{q} X_{1}\left(\frac{l}{l_{L}}\right) .
\end{aligned}
$$

To simplify this, we find $\overline{p_{L}} \bar{q} \bar{p}+\overline{q_{L}} \bar{q}^{2}=\left(\overline{q_{L}} \bar{p}^{2}+\overline{q_{L}} \bar{q}^{2}\right)=$ $\overline{q_{L}}\left(\bar{p}^{2}+\bar{q}^{2}\right)=\overline{q_{L}}, \overline{p_{L}} \bar{p}^{2}+\overline{q_{L}} \bar{p} \bar{q}=\overline{p_{L}} \bar{p}^{2}+\overline{p_{L}} \bar{q}^{2}=\overline{p_{L}}\left(\bar{p}^{2}+\bar{q}^{2}\right)$ $=\overline{p_{L}}, \overline{p_{L} r_{L}} \bar{q} X_{1} \bar{p}+\overline{r_{L} q_{L}} \bar{q} X_{1} \bar{q}=\overline{p_{L} r_{L}} \bar{q} X_{1} \bar{p}+\overline{r_{L} q_{L}}\left(-\bar{p} X_{1} \bar{p}\right)=$
$\overline{r_{L}}\left(\overline{p_{L}} \bar{q}-\overline{q_{L}} \bar{p}\right) X_{1} \bar{p}=0$, and $\overline{r_{L}}\left(\bar{p} \overline{p_{L}} X_{2} \bar{p}+\bar{p} \overline{q_{L}} X_{2} \bar{q}\right)=\overline{r_{L}}\left(\overline{p_{L}}\right.$ $\left.\bar{p} X_{2} \bar{p}+\overline{p_{L}} \bar{q} X_{2} \bar{q}\right)=\overline{r_{L} p_{L}}\left(\bar{p} X_{2} \bar{p}+\bar{q} X_{2} \bar{q}\right)=0$. Under these simplifications, we get

$$
\begin{aligned}
\left\langle\nabla_{e_{1}} e_{2}, v_{L}\right\rangle_{L}= & \overline{q_{L}} X_{1} \overline{r_{L}}-\overline{p_{L}} X_{2} \overline{r_{L}}+\frac{1}{2 \sqrt{L}}\left(\bar{p}_{L}^{2}-\bar{q}_{L}^{2}\right)+\frac{\sqrt{L}}{2}+\frac{{\overline{r_{L}}}^{2}}{2 \sqrt{L}}\left(\bar{p}^{2}-\bar{q}^{2}\right) \\
& +\overline{r_{L}} \bar{p} X_{2}\left(\frac{l}{l_{L}}\right)-\overline{r_{L}} \bar{q} X_{1}\left(\frac{l}{l_{L}}\right)=\frac{l}{l_{L}}\left\langle e_{1}, \nabla_{H} \overline{r_{L}}\right\rangle_{L} \\
& -\overline{r_{L}}\left\langle e_{1}, \nabla_{H}\left(\frac{l}{l_{L}}\right)\right\rangle_{L}+\frac{1}{2 \sqrt{L}}\left(\bar{p}_{L}^{2}-\bar{q}_{L}^{2}\right)+\frac{\sqrt{L}}{2}+\frac{{\overline{r_{L}}}^{2}}{2 \sqrt{L}}\left(\bar{p}^{2}-\bar{q}^{2}\right) .
\end{aligned}
$$

Finally, we use the identity $\left(\left(l / l_{L}\right)-\left(l_{L} / l\right)\right) \nabla_{H} \overline{r_{L}}=\overline{r_{L}} \nabla_{H}\left(l / l_{L}\right)$ in the above equation:

$$
\begin{aligned}
\left\langle\nabla_{e_{1}} e_{2}, v_{L}\right\rangle_{L}= & \frac{l_{L}}{l}\left\langle e_{1}, \nabla_{H} \overline{r_{L}}\right\rangle_{L}+\frac{1}{2 \sqrt{L}}\left(\bar{p}_{L}^{2}-\bar{q}_{L}^{2}\right) \\
& +\frac{\sqrt{L}}{2}+\frac{{\overline{r_{L}}}^{2}}{2 \sqrt{L}}\left(\bar{p}^{2}-\bar{q}^{2}\right) .
\end{aligned}
$$

$$
\begin{aligned}
h_{12}= & h_{21}=-\left\langle\nabla_{e_{1}} e_{2}, v_{L}\right\rangle_{L}=-\frac{l_{L}}{l}\left\langle e_{1}, \nabla_{H} \overline{r_{L}}\right\rangle_{L} \\
& -\frac{1}{2 \sqrt{L}}\left(\bar{p}_{L}^{2}-\bar{q}_{L}^{2}\right)-\frac{\sqrt{L}}{2}-\frac{\bar{r}_{L}^{2}}{2 \sqrt{L}}\left(\bar{p}^{2}-\bar{q}^{2}\right) .
\end{aligned}
$$

Since $\left\langle\nabla_{e_{2}} v_{L}, e_{2}\right\rangle_{L}=-\left\langle\nabla_{e_{2}} e_{2}, v_{L}\right\rangle_{L}$, using the definition of connection, identities in (5), and grouping terms, we have

Therefore,

$$
\begin{aligned}
& \nabla_{e_{2}}^{L} e_{2}=\nabla \frac{L}{r_{L}} \bar{p} X_{1}+\overline{r_{L}} \bar{q} X_{2}-\left(l / l_{L}\right) L^{-(1 / 2)} X_{3}\left(\overline{r_{L}} \bar{p} X_{1}+\overline{r_{L}} \bar{q} X_{2}-\frac{l}{l_{L}} L^{-(1 / 2)} X_{3}\right) \\
& =\left[\overline{r_{L}} \bar{p} X_{1} \overline{r_{L}} \bar{p}+\overline{r_{L}} \bar{q} X_{2} \overline{r_{L}} \bar{p}-\frac{l}{\sqrt{L} l_{L}} X_{3} \overline{r_{L}} \bar{p}-\frac{l \sqrt{L}}{l_{L}} \overline{r_{L}} \bar{q}\right] X_{1} \\
& +\left[\overline{r_{L}} \bar{p} X_{1} \overline{r_{L}} \bar{q}+\overline{r_{L}} \bar{q} X_{2} \overline{r_{L}} \bar{q}-\frac{l}{\sqrt{L} l_{L}} X_{3} \overline{r_{L}} \bar{q}-\frac{\sqrt{r_{L}} \bar{p}}{\sqrt{L} l_{L}}(1-L)\right] X_{2} \\
& +\left(-\frac{\overline{r_{L}} \bar{p}}{\sqrt{L}} X_{1} \frac{l}{l_{L}}-\frac{\overline{r_{L}} \bar{q}}{\sqrt{L}} X_{2} \frac{l}{l_{L}}+\frac{l}{l_{L} L} X_{3} \frac{l}{l_{L}}-\frac{{\overline{r_{L}}}^{2} \bar{p} \bar{q}}{L}\right) X_{3} .
\end{aligned}
$$

Taking the inner product with $v_{L}$ yields 


$$
\begin{aligned}
& \left\langle\nabla_{e_{2}}^{L} e_{2}, v_{L}\right\rangle_{L}={\overline{p_{L} r_{L}}}^{2} X_{1} \overline{r_{L}}+{\overline{p_{L} r_{L}}}^{2} \bar{p} X_{1} \bar{p}+{\overline{p_{L} r_{L}}}_{\bar{q}} \bar{p} X_{2} \overline{r_{L}}+{\overline{p_{L} r_{L}}}^{2} \bar{q} X_{2} \bar{p} \\
& -\overline{p_{L}} \frac{l}{\sqrt{L} l_{L}} \bar{p} X_{3} \overline{r_{L}}-\overline{p_{L}} \frac{l}{\sqrt{L} l_{L}} \overline{r_{L}} X_{3} \bar{p}-\overline{p_{L}} \frac{l \sqrt{L}}{l_{L}} \overline{r_{L}} \bar{q} \\
& +\overline{q_{L} r_{L}} \bar{p} \bar{q} X_{1} \overline{r_{L}}+{\overline{q_{L} r_{L}}}^{2} \bar{p} X_{1} \bar{q}+{\overline{q_{L} r_{L}}}^{2} \bar{q} X_{2} \overline{r_{L}}+{\overline{q_{L} r_{L}}}^{2} \bar{q} X_{2} \bar{q} \\
& -\frac{l \overline{q_{L}}}{l_{L} \sqrt{L}} X_{3} \overline{r_{L}}-\frac{\bar{q} l \overline{r_{L}}}{l_{L} \sqrt{L}} X_{3} \bar{q}-\frac{\overline{q_{L}} l \overline{r_{L}} \bar{p}}{l_{L} \sqrt{L}}(1-L) \\
& +\overline{r_{L}} \sqrt{L}\left(-\frac{\bar{p} \overline{r_{L}}}{\sqrt{L}} X_{1} \frac{l}{l_{L}}-\frac{\bar{q} \overline{r_{L}}}{\sqrt{L}} X_{2} \frac{l}{l_{L}}+\frac{l}{l_{L} L} X_{3} \frac{l}{l_{L}}-\frac{{\overline{r_{L}}}^{2} \bar{p} \bar{q}}{L}\right) .
\end{aligned}
$$

Under some similar simplifications to Theorem 4.3 in [8], we get

$$
h_{22}=-\left\langle\nabla_{e_{2}}^{L} e_{2}, v_{L}\right\rangle_{L}=-\frac{l^{2}}{l_{L}^{2}}\left\langle e_{2}, \nabla_{H}^{L}\left(\frac{r}{l}\right)\right\rangle_{L}+\widetilde{X_{3}}\left(\overline{r_{L}}\right)+\frac{1}{\sqrt{L}} \overline{p_{L} q_{L} r_{L}}+\frac{1}{\sqrt{L}} \bar{p} \overline{q_{L}}{ }^{3} .
$$

The Riemannian mean curvature $\mathscr{H}_{L}$ of $\Sigma$ is defined by

$$
\begin{aligned}
\mathscr{H}_{L}:= & \operatorname{tr}\left(I I^{L}\right)=\frac{l}{l_{L}}\left(X_{1}(\bar{p})+X_{2}(\bar{q})\right)-\overline{r_{L}} \frac{\bar{p} \bar{q}}{\sqrt{L}}-\frac{l^{2}}{l_{L}^{2}}\left\langle e_{2}, \nabla_{H}^{L}\left(\frac{r}{l}\right)\right\rangle_{L} \\
& +\widetilde{X_{3}}\left(\overline{r_{L}}\right)+\frac{1}{\sqrt{L}} \overline{p_{L} q_{L} r_{L}}+\frac{1}{\sqrt{L}} \bar{p} \overline{q_{L}} \bar{r}^{3} .
\end{aligned}
$$

Similar to Proposition 3.8 in [15], away from the characteristic point, the horizontal mean curvature $\mathscr{H}_{\infty}$ of $\Sigma \in\left(\mathscr{R} \mathscr{T}, g_{L}\right)$ is given by

$$
\mathscr{H}_{\infty}=\lim _{L \longrightarrow \infty} \mathscr{H}_{L}=X_{1}(\bar{p})+X_{2}(\bar{q}) .
$$

Let

$$
\begin{array}{r}
\mathscr{K}^{\Sigma, L}\left(e_{1}, e_{2}\right)=\left\langle-R^{\Sigma, L}\left(e_{1}, e_{2}\right) e_{1}, e_{2}\right\rangle_{\Sigma, L} \\
\mathscr{K}^{L}\left(e_{1}, e_{2}\right)=-\left\langle R^{L}\left(e_{1}, e_{2}\right) e_{1}, e_{2}\right\rangle_{L} .
\end{array}
$$

By the Gauss equation, we have

$$
\mathscr{K}^{\Sigma, L}\left(e_{1}, e_{2}\right)=\mathscr{K}^{L}\left(e_{1}, e_{2}\right)+\operatorname{det}\left(I I^{L}\right) .
$$

Proposition 7. Away from characteristic points, we have

$$
\mathscr{K}^{\Sigma, \infty}\left(e_{1}, e_{2}\right)=-\left\langle e_{1}, \nabla_{H}\left(\frac{X_{3} u}{\left|\nabla_{H} u\right|}\right)\right\rangle-\frac{\left(X_{3} u\right)^{2}}{l^{2}} \text { as } L \longrightarrow+\infty .
$$

Proof. We compute 


$$
\begin{aligned}
& R^{L}\left(e_{1}, e_{2}\right) e_{1}=R^{L}\left(\bar{q} X_{1}-\bar{p} X_{2}, \bar{r}_{L} \bar{p} X_{1}+\bar{r}_{L} \bar{q} X_{2}-\frac{l}{l_{L} \sqrt{L}} X_{3}\right)\left(\bar{q} X_{1}-\bar{p} X_{2}\right) \\
& =\bar{r}_{L} \bar{p} \bar{q}^{2} R^{L}\left(X_{1}, X_{1}\right) X_{1}+\bar{r}_{L} \bar{q}^{3} R^{L}\left(X_{1}, X_{2}\right) X_{1}-\frac{l \bar{q}^{2}}{l_{L} \sqrt{L}} R^{L}\left(X_{1}, X_{3}\right) X_{1} \\
& -\bar{r}_{L} \bar{p}^{2} \bar{q} R^{L}\left(X_{2}, X_{1}\right) X_{1}-\bar{r}_{L} \bar{p} \bar{q}^{2} R^{L}\left(X_{2}, X_{2}\right) X_{1}+\frac{l \bar{p} \bar{q}}{l_{L} \sqrt{L}} R^{L}\left(X_{2}, X_{3}\right) X_{1} \\
& -\bar{r}_{L} \bar{p}^{2} \bar{q} R^{L}\left(X_{1}, X_{1}\right) X_{2}-\bar{r}_{L} \bar{p} \bar{q}^{2} R^{L}\left(X_{1}, X_{2}\right) X_{2}+\frac{l \bar{p} \bar{q}}{l_{L} \sqrt{L}} R^{L}\left(X_{1}, X_{3}\right) X_{2} \\
& +\bar{r}_{L} \bar{p}^{3} R^{L}\left(X_{2}, X_{1}\right) X_{2}+\bar{r}_{L} \bar{p}^{2} \bar{q} R^{L}\left(X_{2}, X_{2}\right) X_{2}-\frac{l \bar{p}^{2}}{l_{L} \sqrt{L}} R^{L}\left(X_{2}, X_{3}\right) X_{2} \\
& =\overline{r_{L}} \bar{q} R^{L}\left(X_{1}, X_{2}\right) X_{1}-\frac{l \bar{q}}{l_{L} \sqrt{L}} R^{L}\left(X_{1}, X_{3}\right) X_{1}+\frac{l \bar{p} \bar{q}}{l_{L} \sqrt{L}} R^{L}\left(X_{2}, X_{3}\right) X_{1} \\
& -\overline{r_{L}} \bar{p} R^{L}\left(X_{1}, X_{2}\right) X_{2}+\frac{l \bar{p} \bar{q}}{l_{L} \sqrt{L}} R^{L}\left(X_{1}, X_{3}\right) X_{2}-\frac{l \bar{p}^{2}}{l_{L} \sqrt{L}} R^{L}\left(X_{2}, X_{3}\right) X_{2} \\
& =-\frac{3 L^{2}+2 L+1}{4 L} \bar{r}_{L} \bar{p} X_{1}+\overline{r_{L}} \bar{q} \frac{3 L^{2}-2 L-1}{4 L} X_{2}+\left[\frac{\bar{p}^{2} l}{l_{L} \sqrt{L}} \frac{-L^{2}-2 L-1}{4 L}+\frac{l \bar{p}^{2}}{l_{L} \sqrt{L}} \frac{-L^{2}-2 L+3}{4 L}\right] X_{3} \text {, } \\
& \mathscr{K}^{L}\left(e_{1}, e_{2}\right)=-\left\langle R^{L}\left(e_{1}, e_{2}\right) e_{1}, e_{2}\right\rangle_{L}=-\left(\frac{3}{4} L+\frac{1}{2}+\frac{1}{4 L}\right) \bar{r}_{L}^{2} \bar{p}^{2}-\bar{r}_{L}^{2} \bar{q}^{2}\left(\frac{3}{4} L-\frac{1}{2}-\frac{1}{4 L}\right) \\
& -\bar{p}^{2}\left(\frac{l}{l_{L}}\right)^{2}\left(-\frac{L}{4}-\frac{1}{2}+\frac{3}{4 L}\right)-\frac{l^{2} \bar{q}^{2}}{l_{L}^{2}}\left(-\frac{L}{4}+\frac{1}{2}-\frac{1}{4 L}\right) .
\end{aligned}
$$

To simplify this, we find $-(3 / 4) L \bar{r}_{L}^{2} \bar{p}^{2}-(3$ 14) $L \bar{r}_{L}^{2} \bar{q}^{2}=-(3 / 4) L \bar{r}_{L}^{2} \sim-(3 / 4)\left(\left(X_{3} u\right)^{2} / l^{2}\right)$ as $L \longrightarrow \infty$, $\bar{p}^{2}\left(l / l_{L}\right)^{2}(L / 4)+\bar{q}^{2}\left(l / l_{L}\right)^{2}(L / 4)=\left(l / l_{L}\right)^{2}(L / 4)$, and $\bar{p}^{2}\left(l / l_{L}\right)^{2}$ $(1 / 2)-\bar{q}^{2}\left(l / l_{L}\right)^{2}(1 / 2)=((1 / 2)-\bar{q} 2)\left(l / l_{L}\right)^{2},\left(l / l_{L}\right)^{2} \sim 1$ as $L \longrightarrow \infty$. Finally, we get

$$
\mathscr{K}^{L}\left(e_{1}, e_{2}\right) \sim-\frac{3}{4} \frac{\left(X_{3} u\right)^{2}}{l^{2}}+\frac{L}{4}\left(\frac{l}{l_{L}}\right)^{2}+\frac{1}{2}-\bar{q}^{2} \text { as } L \longrightarrow \infty .
$$

By Theorem 1 and $\nabla_{H}\left(\bar{r}_{L}\right)=L^{-(1 / 2)} \nabla_{H}\left(X_{3} u /\left|\nabla_{H} u\right|\right)+$ $O\left(L^{-1}\right)$ as $L \longrightarrow+\infty$, we get

$$
\operatorname{det}\left(I I^{L}\right)=h_{11} h_{22}-h_{12}^{2}=-\frac{L}{4}-\left\langle e_{1}, \nabla_{H}\left(\frac{X_{3} u}{\left|\nabla_{H} u\right|}\right)\right\rangle+\frac{1}{2}\left(\bar{q}^{2}-\bar{p}^{2}\right)+O\left(L^{-(1 / 2)}\right)
$$

as $L \longrightarrow+\infty$. Here, we have used the equation $(L / 4)\left(l / l_{L}\right)^{2}-(L / 4)=-(L / 4)\left(l_{L}^{2}-l^{2} / l_{L}^{2}\right)=-(L / 4)\left(r^{2} / l_{L}^{2}\right)=$ $-\left(L / 4 l_{L}^{2}\right)\left(\left(X_{3} u\right)^{2} / L\right)=-\left(\left(X_{3} u\right)^{2} / 4 l_{L}^{2}\right) \sim-\left(\left(X_{3} u\right)^{2} / 4 l^{2}\right)$ as $L \longrightarrow+\infty$. By (93), (96), and (97), we get the desired equation.

\section{A Gauss-Bonnet Theorem in the Rototranslation Group}

In this section, we will prove the Gauss-Bonnet theorem in the rototranslation group. Firstly, we consider the case of a regular curve $\gamma:[a, b] \longrightarrow\left(\mathscr{R} \mathscr{T}, g_{L}\right)$. We define the Riemannian length measure

$$
\mathrm{d} s_{L}=\|\dot{\gamma}\|_{L} \mathrm{~d} t
$$

Lemma 1. Let $\gamma:[a, b] \longrightarrow\left(\mathscr{R T}, g_{L}\right)$ be a Euclidean $C^{2}$-smooth and regular curve. Let

$$
\begin{aligned}
\mathrm{d} s & :=|\omega(\dot{\gamma}(t))| \mathrm{d} t \\
\mathrm{~d} \bar{s} & :=\frac{1}{2} \frac{1}{|\omega(\dot{\gamma}(t))|}\left[\left(\dot{\gamma}_{1}(t) \cos \theta+\dot{\gamma}_{2}(t) \sin \theta\right)^{2}+(\dot{\theta}(t))^{2}\right] \mathrm{d} t .
\end{aligned}
$$

Then, 


$$
\lim _{L \longrightarrow \infty} \frac{1}{\sqrt{L}} \int_{\gamma} \mathrm{d} s_{L}=\int_{a}^{b} \mathrm{~d} s .
$$

$$
\frac{1}{\sqrt{L}} \mathrm{~d} s_{L}=\frac{1}{\sqrt{L}} \sqrt{\left(\dot{\gamma}_{1}(t) \cos \theta+\dot{\gamma}_{2}(t) \sin \theta\right)^{2}+(\dot{\theta}(t))^{2}} \mathrm{~d} t .
$$

When $\omega(\dot{\gamma}(t)) \neq 0$, we have

$$
\frac{1}{\sqrt{L}} \mathrm{~d} s_{L}=\mathrm{d} s+\mathrm{d} \bar{s} L^{-1}+O\left(L^{-2}\right) \text { as } L \longrightarrow+\infty \text {. }
$$

When $\omega(\dot{\gamma}(t))=0$, we have

\section{Proof. Since}

$$
\|\dot{\gamma}(t)\|_{L}=\sqrt{\left(\dot{\gamma}_{1}(t) \cos \theta+\dot{\gamma}_{2}(t) \sin \theta\right)^{2}+(\dot{\theta}(t))^{2}+L(\omega(\dot{\gamma}(t)))^{2}},
$$

similar to the proof of Lemma 6.1 in [5], we can prove

$$
\begin{aligned}
\lim _{L \longrightarrow \infty} \frac{1}{\sqrt{L}} \int_{\gamma}\|\dot{\gamma}(t)\|_{L} \mathrm{~d} t & =\int_{a}^{b} \lim _{L \longrightarrow \infty} \frac{1}{\sqrt{L}}\|\dot{\gamma}(t)\|_{L} \mathrm{~d} t \\
& =\int_{a}^{b} \lim _{L \longrightarrow \infty} \frac{1}{\sqrt{L}} \sqrt{\left(\dot{\gamma}_{1}(t) \cos \theta+\dot{\gamma}_{2}(t) \sin \theta\right)^{2}+(\dot{\theta}(t))^{2}+L(\omega(\dot{\gamma}(t)))^{2}} \mathrm{~d} t \\
& =\int_{a}^{b}|\omega(\dot{\gamma}(t))| \mathrm{d} t=\int_{a}^{b} \mathrm{~d} s .
\end{aligned}
$$

When $\omega(\dot{\gamma}(t)) \neq 0$, we have

$$
\frac{1}{\sqrt{L}} \mathrm{~d} s_{L}=\sqrt{L^{-1}\left(\left(\dot{\gamma}_{1}(t) \cos \theta+\dot{\gamma}_{2}(t) \sin \theta\right)^{2}+(\dot{\theta}(t))^{2}\right)+\omega(\dot{\gamma}(t))^{2}} \mathrm{~d} t .
$$

Using the Taylor expansion, we can prove

$\frac{1}{\sqrt{L}} \mathrm{~d} s_{L}=\mathrm{d} s+\mathrm{d} \bar{s} L^{-1}+O\left(L^{-2}\right)$ as $L \longrightarrow+\infty$.

From the definition of $d s_{L}$ and $\omega(\dot{\gamma}(t))=0$, we get $\frac{1}{\sqrt{L}} \mathrm{~d} s_{L}=\frac{1}{\sqrt{L}} \sqrt{\left(\dot{\gamma}_{1}(t) \cos \theta+\dot{\gamma}_{2}(t) \sin \theta\right)^{2}+(\dot{\theta}(t))^{2}} \mathrm{~d} t$.

Proposition 8. Let $\Sigma \subset\left(\mathscr{R} \mathscr{T}, g_{L}\right)$ be a Euclidean $C^{2}$-smooth surface, $\Sigma=\{u=0\}$, and $d \sigma_{\Sigma, L}$ denote the surface measure on $\Sigma$ with respect to the Riemannian metric $g_{L}$. Let

$$
\mathrm{d} \sigma_{\Sigma}:=\left(\bar{p} \omega_{2}-\bar{q} \omega_{1}\right) \wedge \omega
$$

$$
\mathrm{d} \bar{\sigma}_{\Sigma}:=\frac{X_{3} u}{l} \omega_{1} \wedge \omega_{2}-\frac{\left(X_{3} u\right)^{2}}{2 l^{2}}\left(\bar{p} \omega_{2}-\bar{q} \omega_{1}\right) \wedge \omega .
$$

Then,

$$
\frac{1}{\sqrt{L}} \mathrm{~d} \sigma_{\Sigma, L}=\mathrm{d} \sigma_{\Sigma}+\mathrm{d} \bar{\sigma}_{\Sigma} L^{-1}+O\left(L^{-2}\right) \text {, as } L \longrightarrow+\infty \text {. }
$$

If $\Sigma=f(D)$ with $f=f\left(u_{1}, u_{2}\right)=\left(f_{1}, f_{2}, f_{3}\right)$ : $D \subset \mathbb{R}^{2} \longrightarrow\left(\mathscr{R T}, g_{L}\right)$, then

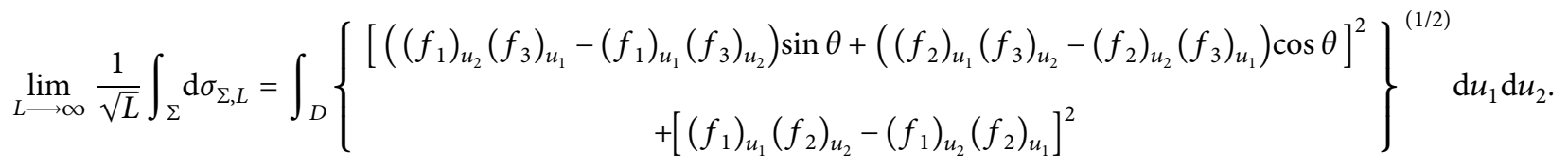


Proof. It is well known that

$$
\begin{aligned}
& g_{L}\left(X_{1}, \cdot\right)=\omega_{1}, \\
& g_{L}\left(X_{2}, \cdot\right)=\omega_{2}, \\
& g_{L}\left(X_{3}, \cdot\right)=L \omega .
\end{aligned}
$$

We define $e_{1}^{*}:=g_{L}\left(e_{1}, \cdot\right)$ and $e_{2}^{*}:=g_{L}\left(e_{2}, \cdot\right)$; then,

$$
\begin{aligned}
& e_{1}^{*}=\bar{q} \omega_{1}-\bar{p} \omega_{2}, \\
& e_{2}^{*}=\bar{r}_{L} \bar{p} \omega_{1}+\bar{r}_{L} \bar{q} \omega_{2}-\frac{l}{l_{L}} L^{(1 / 2)} \omega .
\end{aligned}
$$

Recalling

$$
\bar{r}_{L}=\frac{\left(X_{3} u\right) L^{-(1 / 2)}}{\sqrt{p^{2}+q^{2}+L^{-1}\left(X_{3} u\right)^{2}}}
$$

and the Taylor expansion

$$
\frac{1}{l_{L}}=\frac{1}{l}-\frac{1}{2 l^{3}}\left(X_{3} u\right)^{2} L^{-1}+O\left(L^{-2}\right) \text { as } L \longrightarrow+\infty,
$$

we get (109). By (5), we have

Therefore,

$\frac{1}{\sqrt{L}} \mathrm{~d} \sigma_{\Sigma, L}=\frac{1}{\sqrt{L}} e_{1}^{*} \wedge e_{2}^{*}=\frac{l}{l_{L}}\left(\bar{p} \omega_{2}-\bar{q} \omega_{1}\right) \wedge \omega+\frac{1}{\sqrt{L}} \bar{r}_{L} \omega_{1} \wedge \omega_{2}$.

$$
\begin{aligned}
f_{u_{1}} & =\left(f_{1}\right)_{u_{1}} \partial x_{1}+\left(f_{2}\right)_{u_{1}} \partial x_{2}+\left(f_{3}\right)_{u_{1}} \partial \theta \\
& =\left(f_{1}\right)_{u_{1}}\left(\cos X_{1}+\sin X_{3}\right)+\left(f_{2}\right)_{u_{1}}\left(\sin X_{1}-\cos X_{3}\right)+\left(f_{3}\right)_{u_{1}} X_{2} \\
& =\left(\left(f_{1}\right)_{u_{1}} \cos \theta+\left(f_{2}\right)_{u_{1}} \sin \theta\right) X_{1}+\left(f_{3}\right)_{u_{1}} X_{2}+\sqrt{L}\left(\left(f_{1}\right)_{u_{1}} \sin \theta-\left(f_{2}\right)_{u_{1}} \cos \theta\right) \widetilde{X_{3}}, \\
f_{u_{2}} & =\left(f_{1}\right)_{u_{2}} \partial x_{1}+\left(f_{2}\right)_{u_{2}} \partial x_{2}+\left(f_{3}\right)_{u_{2}} \partial \theta \\
& =\left(\left(f_{1}\right)_{u_{2}} \cos \theta+\left(f_{2}\right)_{u_{2}} \sin \theta\right) X_{1}+\left(f_{3}\right)_{u_{2}} X_{2}+\sqrt{L}\left(\left(f_{1}\right)_{u_{2}} \sin \theta-\left(f_{2}\right)_{u_{2}} \cos \theta\right) \widetilde{X_{3}} .
\end{aligned}
$$

Let

$$
\begin{aligned}
\bar{v}_{L}= & \left|\begin{array}{ccc}
X_{1} & X_{2} & X_{3} \\
\left(f_{1}\right)_{u_{1}} \cos \theta+\left(f_{2}\right)_{u_{1}} \sin \theta & \left(f_{3}\right)_{u_{1}} & \sqrt{L}\left(\left(f_{1}\right)_{u_{1}} \sin \theta-\left(f_{2}\right)_{u_{1}} \cos \theta\right) \\
\left(f_{1}\right)_{u_{2}} \cos \theta+\left(f_{2}\right)_{u_{2}} \sin \theta & \left(f_{3}\right)_{u_{2}} & \sqrt{L}\left(\left(f_{1}\right)_{u_{2}} \sin \theta-\left(f_{2}\right)_{u_{2}} \cos \theta\right),
\end{array}\right| \\
= & \sqrt{L}\left[\left(\left(f_{1}\right)_{u_{2}}\left(f_{3}\right)_{u_{1}}-\left(f_{1}\right)_{u_{1}}\left(f_{3}\right)_{u_{2}}\right) \sin \theta+\left(\left(f_{2}\right)_{u_{1}}\left(f_{3}\right)_{u_{2}}-\left(f_{2}\right)_{u_{2}}\left(f_{3}\right)_{u_{1}}\right) \cos \theta\right] X_{1} \\
& +\sqrt{L}\left(\left(f_{1}\right)_{u_{1}}\left(f_{2}\right)_{u_{2}}-\left(f_{1}\right)_{u_{2}}\left(f_{2}\right)_{u_{1}}\right) X_{2} \\
& +\left[\left(\left(f_{1}\right)_{u_{1}}\left(f_{3}\right)_{u_{2}}-\left(f_{1}\right)_{u_{2}}\left(f_{3}\right)_{u_{1}}\right) \cos \theta+\left(\left(f_{2}\right)_{u_{1}}\left(f_{3}\right)_{u_{2}}-\left(f_{2}\right)_{u_{2}}\left(f_{3}\right)_{u_{1}}\right) \sin \theta\right] \widetilde{X_{3}} .
\end{aligned}
$$

We know that $\mathrm{d} \sigma_{\Sigma, L}=\sqrt{\operatorname{det}\left(g_{i j}\right)} \mathrm{d} u_{1} \mathrm{~d} u_{2}, g_{i j}=g_{L}\left(f_{u_{i}}\right.$, $\left.f_{u_{j}}\right)$, and

$$
\begin{aligned}
\operatorname{det}\left(g_{i j}\right)= & \left\|\bar{v}_{L}\right\|_{L}^{2}=L\left[\left(\left(f_{1}\right)_{u_{2}}\left(f_{3}\right)_{u_{1}}-\left(f_{1}\right)_{u_{1}}\left(f_{3}\right)_{u_{2}}\right) \sin \theta+\left(\left(f_{2}\right)_{u_{1}}\left(f_{3}\right)_{u_{2}}-\left(f_{2}\right)_{u_{2}}\left(f_{3}\right)_{u_{1}}\right) \cos \theta\right]^{2} \\
& +L\left[\left(f_{1}\right)_{u_{1}}\left(f_{2}\right)_{u_{2}}-\left(f_{1}\right)_{u_{2}}\left(f_{2}\right)_{u_{1}}\right]^{2}+\left[\left(\left(f_{1}\right)_{u_{1}}\left(f_{3}\right)_{u_{2}}-\left(f_{1}\right)_{u_{2}}\left(f_{3}\right)_{u_{1}}\right) \cos \theta+\left(\left(f_{2}\right)_{u_{1}}\left(f_{3}\right)_{u_{2}}-\left(f_{2}\right)_{u_{2}}\left(f_{3}\right)_{u_{1}}\right) \sin \theta\right]^{2},
\end{aligned}
$$

so by the dominated convergence theorem, we get 


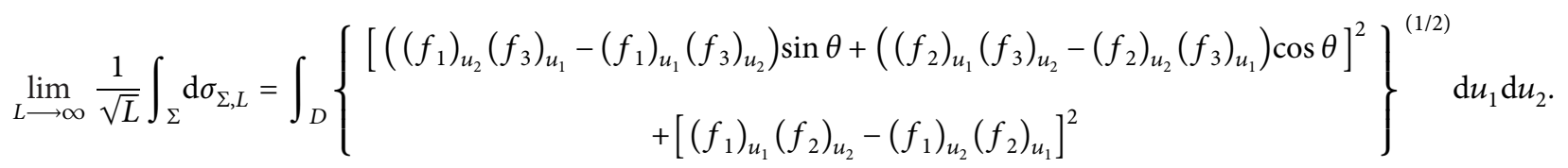

Similar to the proof of Theorem 4.3 in [6], we get a Gauss-Bonnet theorem in the rototranslation group as follows.

Theorem 2. Let $\Sigma \subset\left(\mathscr{R} \mathscr{T}, g_{L}\right)$ be a regular surface with finitely many boundary components $(\partial \Sigma)_{i}, i \in\{1, \ldots, n\}$, given by Euclidean $C^{2}$-smooth regular and closed curves $\gamma_{i}:[0,2 \pi] \longrightarrow(\partial \Sigma)_{i}$. Suppose that the characteristic set $C(\Sigma)$ satisfies $\mathscr{H}^{1}(C(\Sigma))=0$ where $\mathscr{H}^{1}(C(\Sigma))$ denotes the Euclidean 1-dimensional Hausdorff measure of $C(\Sigma)$ and that $\left\|\nabla_{H} u\right\|_{H}^{-1}$ is locally summable with respect to the Euclidean 2-dimensional Hausdorff measure near the characteristic set $C(\Sigma)$; then,

$$
\int_{\Sigma} \mathscr{K}^{\Sigma, \infty} \mathrm{d} \sigma_{\Sigma}+\sum_{i=1}^{n} \int_{\gamma_{i}} \kappa_{\gamma_{i}, \Sigma}^{\infty, s} \mathrm{~d} s=0
$$

Proof. Using the similar discussions in $[13,14]$, we assume that all points satisfy $\omega\left(\dot{\gamma}_{i}(t)\right) \neq 0$ on the curve $\gamma_{i}$. Recalling the result in Proposition 4 indicates

$$
\kappa_{\gamma_{i}, \Sigma}^{L, s}=\kappa_{\gamma_{i}, \Sigma}^{\infty, s}+O\left(L^{-(1 / 2)}\right)
$$

According to the Gauss-Bonnet theorem, we get

$$
\int_{\Sigma} \mathscr{K}^{\Sigma, L} \frac{1}{\sqrt{L}} \mathrm{~d} \sigma_{\Sigma, L}+\sum_{i=1}^{n} \int_{\gamma_{i}} \kappa_{\gamma_{i}, \Sigma}^{L, s} \frac{1}{\sqrt{L}} \mathrm{~d} s_{L}=2 \pi \frac{\chi(\Sigma)}{\sqrt{L}} .
$$

Let $L$ go to the infinity, and using the dominated convergence theorem, (121), (122), (109), Proposition 6, and Lemma 1 , we get the desired result.

\section{Conclusion}

This paper dealt with an interesting question of the GaussBonnet theorem in the rototranslation group from the Riemannian approximation scheme. The main result of this paper is Theorem 2, which is the Gauss-Bonnet-type theorem in the rototranslation group. To prove Theorem 2, we obtained the sub-Riemannian limit of the curvature of curves, sub-Riemannian limits of the geodesic curvature of curves on surfaces, and the Riemannian Gaussian curvature of surfaces in the rototranslation group.

As a future work, we plan to proceed to study Gauss-Bonnet theorems in the rototranslation group with the general left-invariant metric and other three-dimensional Riemannian Lie groups which were classified in [18]. In these conditions, Gauss-Bonnet theorems can be obtained through the Riemannian approximation scheme took by Balogh et al. $[13,14]$. The Gauss-Bonnet theorem connects the intrinsic differential geometry of a surface with its topology and has many applications. Therefore, it will be interesting to extend the Gauss-Bonnet theorem to other different Lie groups. We believe that the results to be obtained will have some geometric applications.

\section{Data Availability}

No data were used to support this study.

\section{Conflicts of Interest}

The authors declare that there are no conflicts of interest in this work.

\section{Authors' Contributions}

All the authors contributed equally to the writing of this paper and read and approved the final manuscript.

\section{Acknowledgments}

This research was funded by the Reform and Development Foundation for Local Colleges and Universities of the Central Government (Excellent Young Talents Project of Heilongjiang Province, Grant no. ZYQN2019071) and the Project of Science and Technology of Heilongjiang Provincial Education Department (Grant nos. 1355MSYYB005 and 1354ZD008).

\section{References}

[1] A. O. Petters, "Multiplane gravitational lensing. II. Global geometry of caustics," Journal of Mathematical Physics, vol. 36, no. 8, pp. 4276-4295, 1995.

[2] G. W. Gibbons and M. C. Werner, "Applications of the GaussBonnet theorem to gravitational lensing," Classical and Quantum Gravity, vol. 25, no. 23, pp. 7199-7200, 2008.

[3] A. Övgün, K. Jusufi, and İ. Sakall, "Gravitational lensing under the effect of Weyl and bumblebee gravities: applications of Gauss-Bonnet theorem," Annals of Physics, vol. 399, pp. 193-203, 2018.

[4] A. Övgün, İ. Sakallı, and J. Saavedra, "Weak gravitational lensing by Kerr-MOG black hole and Gauss-Bonnet theorem," Annals of Physics, vol. 411, Article ID 167978, 2019.

[5] A. Övgün and İ. Sakall,, "Hawking radiation via Gauss-Bonnet theorem," Annals of Physics, vol. 413, Article ID 168071, 2020.

[6] A. Övgün, İ. Sakallı, and J. Saavedra, "Effect of null aether field on weak deflection angle of black holes," Chinese Physics $C$, vol. 44, no. 12, Article ID 125105, 2020.

[7] D. Chen, C. Gao, X. Liu, and C. Yu, "The correspondence between shadows and test fields in four-dimensional charged Einstein-Gauss-Bonnet black holes," 2021, https://arxiv.org/ pdf/2103.03624.pdf.

[8] L. Capogna, D. Danielli, S. Pauls et al., "An introduction to the Heisenberg group and the sub-riemannian isoperimetric 
problem," Progress in Mathematics, Vol. 259, Birkhäuser, Basel, Switzerland, 2007.

[9] R. K. Hladky and S. D. Pauls, "Minimal surfaces in the rototranslation group with applications to a neuro-biological image completion model," Journal of Mathematical Imaging and Vision, vol. 36, no. 1, pp. 1-27, 2010.

[10] K. I. Pappas, "Straight ruled surfaces in the roto-translational group," Masters Thesis, University of Crete, Masters Thesis, University of Crete016.

[11] E. J. Bekkers, M. Loog, B. M. T. H. Romeny, and R. Duits, "Template matching via densities on the roto-translation group," IEEE Transactions on Pattern Analysis and Machine Intelligence, vol. 40, no. 2, pp. 452-466, 2018.

[12] B. Franceschiello, A. Mashtakov, G. Citti, and A. Sarti, "Geometrical optical illusion via sub-Riemannian geodesics in the roto-translation group," Differential Geometry and Its Applications, vol. 65, pp. 55-77, 2019.

[13] Z. M. Balogh, J. T. Tyson, and E. Vecchi, "Intrinsic curvature of curves and surfaces and a Gauss-Bonnet theorem in the Heisenberg group," Mathematische Zeitschrift, vol. 287, no. 12, pp. 1-38, 2017.

[14] Z. M. Balogh, J. T. Tyson, and E. Vecchi, "Correction to: intrinsic curvature of curves and surfaces and a Gauss-Bonnet theorem in the Heisenberg group," Mathematische Zeitschrift, vol. 296, no. 1-2, pp. 875-876, 2020.

[15] Y. Wang and S. Wei, "Gauss-Bonnet theorems in the affine group and the group of rigid motions of the Minkowski plane," Science China Mathematics, vol. 63, pp. 1-18, 2020.

[16] Y. Wang and S. Wei, "Gauss-bonnet theorems in the BCV spaces and the twisted Heisenberg group," Results in Mathematics, vol. 75, no. 126, pp. 1-21, 2020.

[17] S. Wei and Y. Wang, "Gauss-bonnet theorems in the lorentzian Heisenberg group and the lorentzian group of rigid motions of the Minkowski plane," Symmetry, vol. 13, no. 2, pp. 1-30, 2021.

[18] J. Milnor, "Curvatures of left invariant metrics on lie groups," Advances in Mathematics, vol. 21, no. 3, pp. 293-329, 1976. 